ARTICLE

\title{
Genetic fate-mapping reveals surface accumulation but not deep organ invasion of pleural and peritoneal cavity macrophages following injury
}

Hengwei Jin ${ }^{1,11}$, Kuo Liu ${ }^{1,2,11}$, Juan Tang ${ }^{1,11}$, Xiuzhen Huang (10 1, Haixiao Wang ${ }^{1}$, Qianyu Zhang ${ }^{3}$, Huan Zhu (1) 1, Yan Li (1) 1, Wenjuan Pu (1) 1, Huan Zhao (1) 1, Lingjuan He (1) 1, Yi Li (1) 1, Shaohua Zhang ${ }^{1}$ Zhenqian Zhang', Yufei Zhao ${ }^{4}$, Yanqing Qin ${ }^{5}$, Stefan Pflanz ${ }^{6}$, Karim E. I. Kasmi ${ }^{6}$, Weiyi Zhang ${ }^{6}$, Zhaoyuan Liu ${ }^{7}$, Florent Ginhoux (iD ${ }^{8}$, Yong Ji id ${ }^{9}$, Ben He ${ }^{10}$, Lixin Wang ${ }^{4}$ \& Bin Zhou (iD ${ }^{1,2,3 凶}$

During injury, monocytes are recruited from the circulation to inflamed tissues and differentiate locally into mature macrophages, with prior reports showing that cavity macrophages of the peritoneum and pericardium invade deeply into the respective organs to promote repair. Here we report a dual recombinase-mediated genetic system designed to trace cavity macrophages in vivo by intersectional detection of two characteristic markers. Lineage tracing with this method shows accumulation of cavity macrophages during lung and liver injury on the surface of visceral organs without penetration into the parenchyma. Additional data suggest that these peritoneal or pleural cavity macrophages do not contribute to tissue repair and regeneration. Our in vivo genetic targeting approach thus provides a reliable method to identify and characterize cavity macrophages during their development and in tissue repair and regeneration, and distinguishes these cells from other lineages.

\footnotetext{
${ }^{1}$ State Key Laboratory of Cell Biology, Shanghai Institute of Biochemistry and Cell Biology, Center for Excellence in Molecular Cell Science, Chinese Academy of Sciences, University of Chinese Academy of Sciences, Shanghai, China. ${ }^{2}$ School of Life Science, Hangzhou Institute for Advanced Study, University of Chinese Academy of Sciences, Hangzhou, China. ${ }^{3}$ School of Life Science and Technology, ShanghaiTech University, Shanghai, China. ${ }^{4}$ Department of Cardiac Surgery, Zhongshan Hospital, Fudan University, Shanghai, China. ${ }^{5}$ Shanghai Institute of Nutrition and Health, Chinese Academy of Sciences, Shanghai, China. ${ }^{6}$ Boehringer Ingelheim Pharma GmbH \& Co KG, Biberach an der Riss, Germany. ${ }^{7}$ Shanghai Institute of Immunology, Department of Immunology and Microbiology, Shanghai Jiao Tong University School of Medicine, Shanghai, China. ${ }^{8}$ Singapore Immunology Network, Agency for Science, Technology and Research, Singapore, Singapore. ${ }^{9}$ The Collaborative Innovation Center for Cardiovascular Disease Translational Medicine, Nanjing Medical University, Nanjing, China. ${ }^{10}$ Department of Cardiology, Shanghai Chest Hospital, Shanghai Jiaotong University, Shanghai, China. ${ }^{11}$ These authors contributed equally: Hengwei Jin, Kuo Liu, Juan Tang. ${ }_{\text {email: zhoubin@sibs.ac.cn }}$
} 
M acrophages are found in multiple tissues or organs with great functional diversity during development and during organ homeostasis, immunity, tissue repair, and regeneration ${ }^{1}$. Understanding this diversity and their contribution to pathophysiological processes may provide new therapeutic targets for many human diseases ${ }^{2}$. The long-held belief has been that tissue macrophages in the adult are continuously replenished by bone marrow-derived circulating monocytes ${ }^{3,4}$. In response to tissue injury and inflammation, monocytes from the circulation are recruited to the infected or damaged sites to neutralize and eliminate injurious stimuli ${ }^{5}$. These newly recruited monocytes differentiate into mature macrophages, where they promote tissue remodeling and repair ${ }^{6}$. Recently, a series of elegant lineagetracing studies reveal that many adult tissue macrophages actually originate from resident embryonically-derived early progenitors cells, rather than from circulating monocytes, and that some of these resident macrophages can self-renew locally throughout adult life with minimal contribution from circulating monocytes $^{7-10}$. For example, cardiac resident macrophages are key mediators of cardiac recovery by reducing inflammation and enhancing tissue repair ${ }^{11}$. Further, multiple cardiac macrophage subsets are found in the adult heart that is differentially derived and marked by various functions, including CCR2 expression, which distinguishes peripheral monocyte-derived macrophages from embryonically-derived resident macrophages ${ }^{12-14}$. Subsequent studies revealed that myocardial infarction induced replacement of resident macrophages with those from recruited monocytes, some of which adopted a cell fate nearly indistinguishable from resident macrophages ${ }^{15}$. These studies demonstrate that both circulating monocytes and tissue-resident macrophages are involved during tissue inflammation, repair, and regeneration.

In mammals, the peritoneal, pleural, and pericardial cavities, which are the three major cavities of adults, provide a mechanical buffer as well as lubrication for the movement of visceral organs. Recently, a new paradigm has emerged that proposes that distinct from monocyte-derived or tissue-resident macrophages, mature macrophages can be rapidly recruited from body cavities to the visceral organs, such as the liver and heart, to promote tissue repair after injury 16,17 . This so-called "wormhole migration" of cavity macrophages to visceral organs represents a new model for inflammatory cell recruitment ${ }^{18}$. Cavity macrophages express a high level of GATA6, which distinguishes them from other tissueresident macrophages or circulating monocytes ${ }^{19-21}$. Cell transplantation assays using reporter mice reveal that GATA6 ${ }^{+}$cavity macrophages infiltrate visceral organs via the mesothelium, a single mesothelial cell layer that covers all visceral organs in the cavity ${ }^{16,17}$. Based on Gata6 reporter activity, Kubes' group reported that $\mathrm{GATA}^{+}$cavity macrophages invade deep into the injured liver and heart shortly after the initial insult via a nonvascular route, and many of them are detected greater than 500 $\mu \mathrm{m}$ from the organ surface ${ }^{16,17}$. Depletion of these cavity macrophages by clodronate treatment results in significant weight loss in a $\mathrm{CCl}_{4}$ model of liver fibrosis in mice ${ }^{16}$, while Gata6 gene deletion using a Lyz2-Cre driver impaired the beneficial role of cavity macrophages in ameliorating cardiac fibrosis ${ }^{17}$. These Gata6 reporter analyses and cell transplantation experiments provide the key evidence supporting a new model of inflammatory cell recruitment and contribution to tissue repair and regeneration. These exciting and highly influential findings may represent an entirely new field for macrophage biology and regenerative medicine, while also providing a potential therapeutic strategy to treat organ inflammation and to promote tissue repair. However, unlike the previous tissue-resident macrophage studies that utilized genetic lineage tracing to unravel the discrete origins of these cells, the new paradigm involving the recruitment of cavity macrophages during organ repair still lacks direct genetic lineage tracing evidence to support its conclusions.

Here, we report a novel dual recombinase-mediated genetic system that could be used for specifically labeling the cavity macrophages in vivo. Fate-mapping results show that cavity macrophages accumulate on the surface of the lung and liver without invading deep into the parenchyma after injuries. Furthermore, genetic ablation of cavity macrophages suggests their minimal functional contribution to tissue repair and regeneration. This study provides a specific genetic tool to better understand the precise roles of cavity macrophages in homeostasis, tissue repair, and regeneration.

\section{Results}

Generation of a cavity macrophage-specific mouse reporter for lineage tracing. Specific genetic targeting of endogenous cavity macrophages is critical to understand theirs in vivo cell fate and potential physiological functions in visceral organ inflammation and repair. However, a single genetic marker to distinguish these cells from other cell lineages has yet to be identified. The transcription factor GATA6 has been reported to be selectively expressed in resident cavity macrophages ${ }^{19-22}$ and distinguishes them from other tissue-resident macrophages and monocytes. But while GATA6 is specific for cavity macrophages among macrophage populations, it is also expressed in multiple nonmacrophage cell lineages in various visceral organs, including hepatocytes, cardiomyocytes, lung epithelial cells, mesenchymal stromal cells, and other cell lineages of these organs ${ }^{23-26}$. This wide expression of GATA6 markedly undermines its utility to uniquely trace recruited cavity macrophages into visceral organs.

To allow for specific tracing of endogenous cavity macrophages, we developed a dual recombinase-mediated intersectional genetic lineage tracing system based on Cre-loxP and Dre-rox (Fig. 1a), which are orthogonal in their recombination ${ }^{27-29}$. In our design, the promoter of the gene encoding the hematopoietic cell marker CD45 was used to drive Dre recombinase, while the Gata6 gene promoter was used to drive an inducible CreER (Fig. 1a). In this way, CD45 marker could be used to distinguish macrophages (as they are $\mathrm{CD}_{4}{ }^{+}$) from $\mathrm{CD}_{4} 5^{-}$organ cells (hepatocytes, cardiomyocytes, etc.); Gata6 marker could be used to distinguish cavity macrophages (as they are $\mathrm{Gata6}^{+}$) from recruited monocytes or resident-tissue macrophages (which are $\mathrm{Gata6}^{-}$). Thus, in theory, double-positive cells (i.e., $\mathrm{CD} 45^{+} \mathrm{Gata}^{+}$) would be cavity macrophages and thus be distinguished from tissue-resident macrophages or monocytes, as well as any other cell lineage, such as hepatocytes, cardiomyocytes, or lung epithelial cells (Fig. 1b). To make this system applicable for potential gene manipulation (i.e., knockout or over-expression) to facilitate functional studies, in addition to lineage tracing, we adopted a sequential intersectional genetic approach, by which Dre-mediated activation of CreER allows for subsequent targeting of any available loxP allele ${ }^{30}$. We anticipated that in $\mathrm{CD} 45^{+}$cells, the CD45-Dre driver would remove the rox-flanked transcriptional stop cassette, yielding a Gata6CreER genotype after Dre-rox recombination. Subsequent tamoxifen (Tam) treatment would then enable a controlled CreERmediated recombination event at the R26-tdTomato reporter, leading to constitutive expression of tdTomato in $\mathrm{CD} 45^{+} \mathrm{Gata}^{+}$ cells, and all their descendants, resulting in an indelible genetic marker that would allow for permanent and specific lineage tracing of cavity macrophages (Fig. 1a, b).

We first generated a CD45-Dre knock-in mouse line and crossed it with a strain bearing an R26-rox-tdTomato reporter ${ }^{31}$. We found that CD45-Dre specifically and efficiently targeted hematopoietic cells in the blood and spleen (Supplementary Fig. 1). We next generated Gata6-rox-stop-rox-CreER knock-in 
a

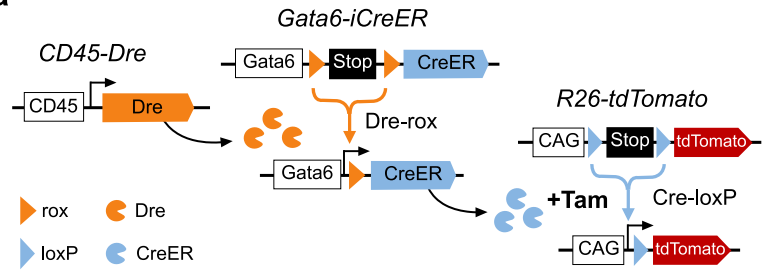

b

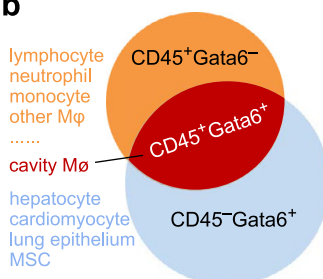

CD45-Dre;Gata6-iCreER

(G6Mø-CreER) R26-tdTomato

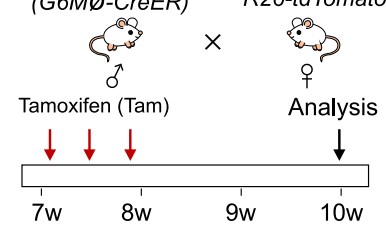

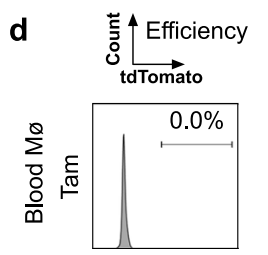

e

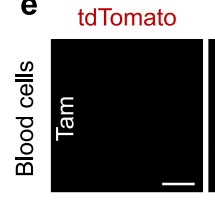

GATA
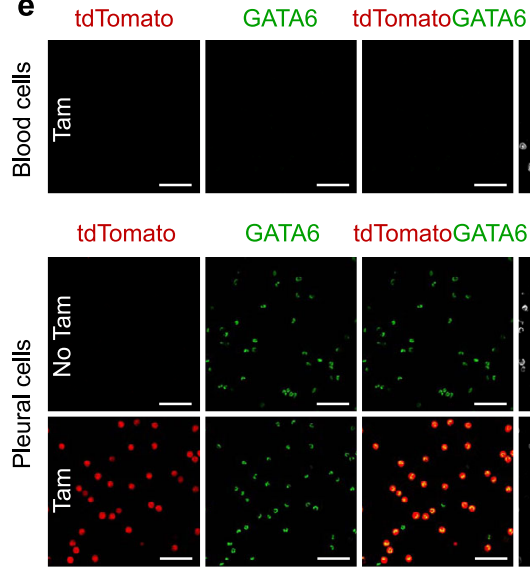

GATA6 tdTomatoGATA6
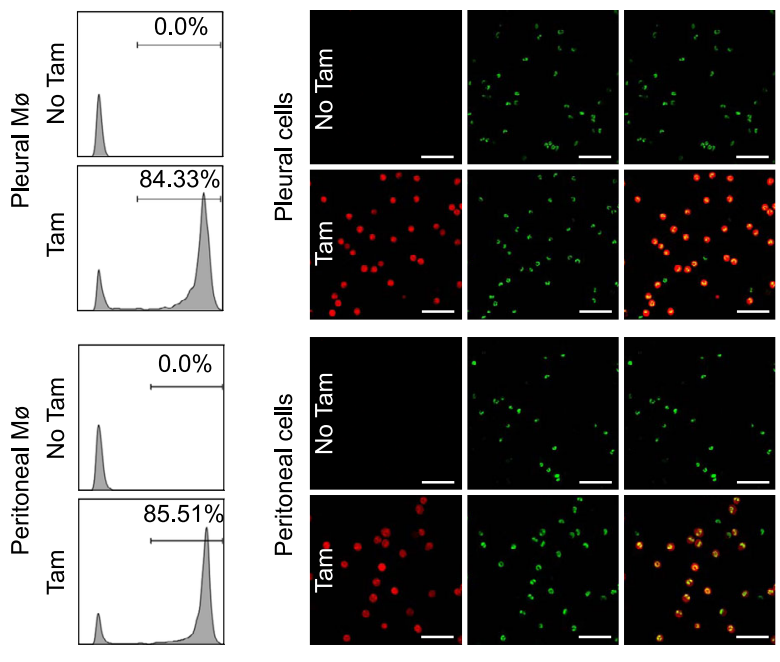
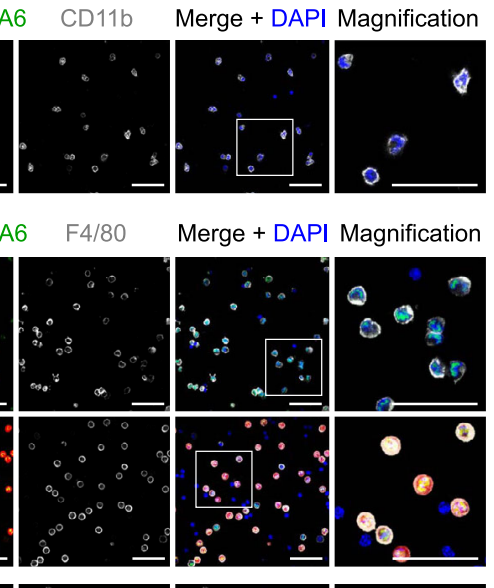

Merge + DAPI Magnification
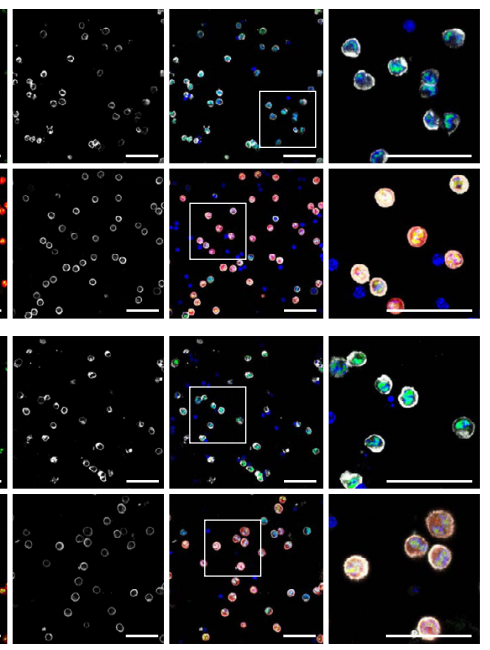

$\mathbf{f}$

Efficiency (Tam)
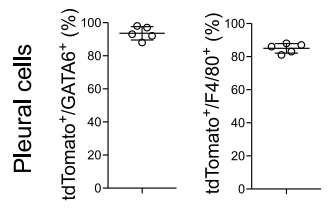

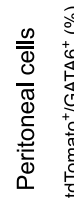

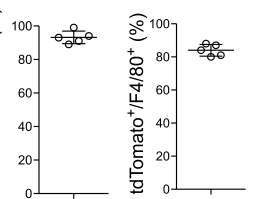

g

\section{Specificity (Tam)}
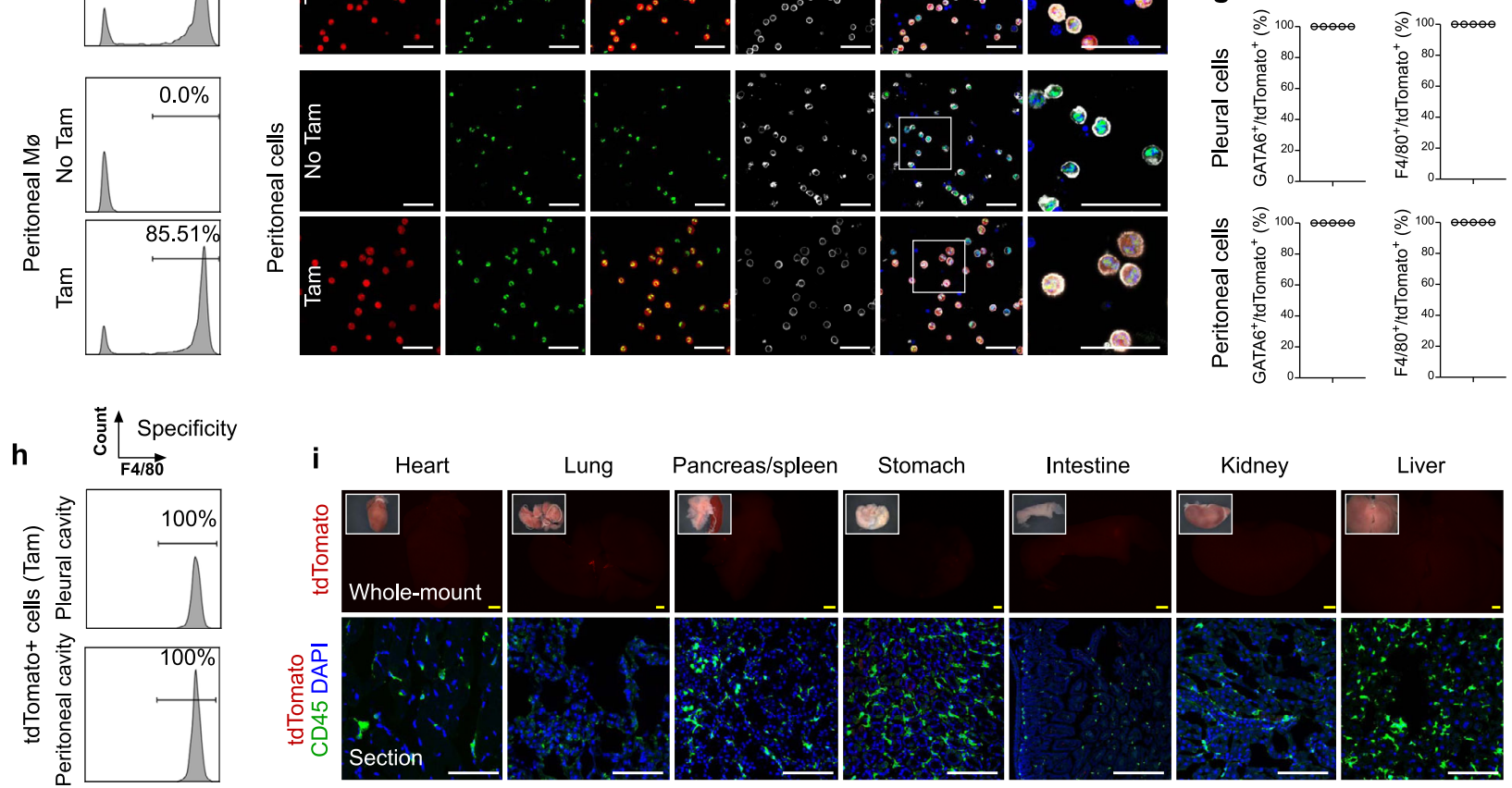

Fig. 1 Generation and characterization of a genetic system for specific targeting of cavity macrophages. a Schematic figure showing CD45-Dre mediates Stop cassette removal from Gata6-iCreER and places CreER directly under Gata6 promoter. After tamoxifen (Tam), Cre-loxP recombination labels cells by tdTomato. $\mathbf{b}$ Intersectional genetics marks $\mathrm{CD} 45^{+} \mathrm{GATA6}{ }^{+}$cells as tdTomato ${ }^{+}$. c Schematic figure showing experimental design using cavity macrophage tracing tool CD45-Dre;Gata6-iCreER (G6Mø-CreER). d Flow cytometric analysis of the percentage of tdTomato ${ }^{+}$cells in macrophages from blood, pleural, and peritoneal cavity with or without Tam. e Immunostaining for tdTomato, CD11b, GATA6, F4/80 on dissociated cells from blood, pleural, or peritoneal cavity. Boxed regions are magnified. $\mathbf{f}$ Quantification of the percentage of tdTomato ${ }^{+}$cells in $\mathrm{GATA6}^{+}$or $\mathrm{F} 4 / 80^{+}$macrophages. Data are the mean $\pm \mathrm{SD}$; $n=5$ mice per group. $\mathbf{g}$ Quantification of the percentage of $\mathrm{GATA6}^{+}$or $\mathrm{F} 4 / 80^{+}$macrophages in tdTomato ${ }^{+}$cells. Data are the mean $\pm \mathrm{SD} ; n=5$ mice per group. h FACS showing the percentage of tdTomato ${ }^{+}$cells expressing F4/80. i Whole-mount epifluorescence images and immunostaining for CD45 and tdTomato shows no resident tdTomato ${ }^{+}$macrophages in visceral organs. Each image is representative of 5 individual biological samples. Scale bars, yellow, $1 \mathrm{~mm}$; white, $100 \mu \mathrm{m}$. Source data are provided as a Source Data file.

mice, a Dre-induced Gata6-CreER (Gata6-iCreER) line, by inserting a rox-stop-rox-CreER cassette into the Gata6 gene locus to replace the endogenous translational start codon (Supplementary Fig. 2a). As there is a transcriptional stop cassette before CreER, Tam treatment alone would not be sufficient to induce tdTomato genetic labeling of GATA6 ${ }^{+}$cavity macrophages (Supplementary Fig. 2b, f). To test whether Gata6iCreER could indeed label GATA6 ${ }^{+}$cells, we generated a Gata6CreER mouse line by removing the transcriptional stop cassette from Gata6-iCreER by using $A C T B-C r e^{32}$. Then by crossing Gata6-CreER with R26-tdTomato reporter mouse ${ }^{33}$, Gata6-CreER efficiently labeled cavity macrophages as tdTomato ${ }^{+}$upon Tam 
treatment (Supplementary Fig. 3a, b). The generation of the inducible reporter line was critical as we found that the use of Gata6-CreER resulted in labeling of diverse cell types in multiple visceral organs, such as hepatocytes, epithelial cells, and cardiomyocytes (Supplementary Fig. 3c, d), which is consistent with previous reports on its broad expression pattern ${ }^{23-26}$. This broad genetic targeting of virtually all visceral organs and tissues excluded the direct use of the Gata6-CreER mouse for the study of cavity macrophages in visceral organ repair. We, therefore, took advantage of CD45-Dre to restrict the targeting domain of Gata6-iCreER in cavity macrophages.

For proof of principle, we crossed CD45-Dre;Gata6-iCreER (G6Mø-CreER) with R26-tdTomato and analyzed monocyte/macrophage labeling at two weeks after Tam treatment (Fig. 1c). FACS analysis and immunofluorescent (IF) staining revealed that the majority of pleural and peritoneal macrophages expressing CD11b and $\mathrm{F} 4 / 80$ were tdTomato ${ }^{+}$, while $\mathrm{CD}_{11 \mathrm{~b}^{+}}$monocytes from blood mononuclear cells were tdTomato ${ }^{-}$in Tam-treated G6Mø-CreER; R26-tdTomato mice (Fig. 1d-f). Virtually all tdTomato ${ }^{+}$pleural and peritoneal cells $(>99.5 \%)$ were GATA6 ${ }^{+} \mathrm{F} 4 / 80^{+}$(Fig. 1g,h), indicating high specificity of $G 6 \mathrm{Mø}-\mathrm{CreER}$ for labeling cavity macrophages. Of note, we did not detect any tdTomato $^{+}$cavity macrophages from G6Mø-CreER;R26-tdTomato mice without Tam treatment (No Tam, Fig. 1d, e), indicating no leakiness of G6Mø-CreER for cavity macrophage labeling. In all examined visceral organs collected from Tam-treated G6Mø-CreER;R26-tdTomato mice, we did not detect tdTomato expression in $\mathrm{CD} 45^{+}$cells, excluding ectopic labeling of resident macrophages or other hematopoietic cell lineages by G6MøCreER in these visceral organs (Fig. 1i). Taken together, the development of the G6Mø-CreER mouse allows for specific and efficient targeting of endogenous cavity macrophages.

Resident cavity macrophages do not invade deep into the liver for its repair. Previous studies have shown that GATA6 ${ }^{+}$cavity macrophages invade deep into the liver after $\mathrm{CCl}_{4^{-}}$or heatinduced injury (HI, induced by touching a heated thermos probe to the liver surface). Further, transplantation of peritoneal macrophages from $L y z M-e G F P$ donor mice into $\mathrm{CCl}_{4}$-injured host mice resulted in $\mathrm{GFP}^{+}$cell migration across the mesothelium and penetration deep into the intrahepatic area ${ }^{16}$. To recapitulate the recruitment and invasion of GATA6 ${ }^{+}$cavity macrophages without a cell transplantation approach, we performed the same injury models of $\mathrm{CCl}_{4}$ treatment and $\mathrm{HI}$, and also included additional cryoinjury (CI) and acetaminophen (APAP) injury models in G6Mø-CreER;R26-tdTomato mice (Fig. 2a and Supplementary Fig. 5a). For sham control, we opened the abdomen and closed it without any further manipulation. One week after Tam injection, mice were challenged with different injury models and livers were collected shortly after treatment as previously indicated $^{16}$. While we could hardly detect any noticeable tdTomato $^{+}$signals from whole-mount fluorescent livers collected from sham, $\mathrm{CCl}_{4^{-}}$and APAP-treated mice, we did observe bright tdTomato $^{+}$regions in the livers from the HI and CI models (Fig. 2b and Supplementary Fig. 5b). Sirius Red staining of liver sections showed a greater fibrotic response in the $\mathrm{CCl}_{4}$ - and APAP-treated groups compared to the Sham control (Fig. 2c and Supplementary Fig. 5c), consistent with previous studies reporting that a single dose of $\mathrm{CCl}_{4}$ or APAP results in severe necroinflammatory injury peaking at day 1 after injury ${ }^{16,34}$. We noticed that in the HI model the injured liver region exhibited thickened mesothelial cell layers that covered the surface of livers, while in the CI model injured liver regions showed pronounced Sirius Red-positive staining, suggestive of severe necrosis (Fig. 2c).

We next examined tdTomato expression in livers of the above injury models. Immunostaining of liver sections for tdTomato,
F4/80, and GATA6 revealed no accumulation of tdTomato ${ }^{+}$ cavity macrophages inside liver parenchymal tissue after $\mathrm{CCl}_{4}$ or APAP treatment (Fig. 2d and Supplementary Fig. 5d), in contrast to previously reported data showing that a substantial number of transplanted LysM-GFP ${ }^{+}$peritoneal cells infiltrated over $500 \mu \mathrm{m}$ into the intrahepatic area ${ }^{16}$. FACS analysis confirmed almost no tdTomato ${ }^{+}$macrophages in Sham, $\mathrm{CCl}_{4}$-or APAP-treated livers (Fig. 2e and Supplementary Fig. 5e). In injured regions of the liver from both the HI and CI models, we did notice an accumulation of tdTomato ${ }^{+}$cavity macrophages, but virtually all of the cells were on the surface of thickened mesothelial layers (m.l.) without any noticeable deep invasion into the liver parenchyma (Fig. 2d). FACS analysis of cells collected from the tdTomato-bright regions (i.e., the injury sites) of the livers from the HI or CI models revealed less than $1 \%$ of macrophages were tdTomato $^{+}$(Fig. 2e). During late phase after injury, immunostaining and FACS analysis of $\mathrm{CCl}_{4}$-treated livers further confirmed tdTomato ${ }^{+}$ macrophages minimally invaded deep into the injured tissues at any detected time points (Supplementary Fig. 6). To further investigate whether transplanted cavity macrophages could invade deep into the liver after injuries, peritoneal macrophages from the G6Mø-CreER;R26-tdTomato mice were collected and transferred to the wild-type mice before $\mathrm{HI}$ - and $\mathrm{CCl}_{4}$-induced injuries. Immunostaining and FACS analysis of injured areas showed no evidence for the migration of tdTomato ${ }^{+}$macrophages into the parenchymal tissues (Supplementary Fig. 7). In addition to the acute injury, we also performed $\mathrm{CCl}_{4}$-induced chronic liver injury on G6Mø-CreER;R26-tdTomato mice, and found cavity macrophages minimally invaded visceral organs for tissue repair and regeneration even in chronic injury models (Supplementary Fig. 8). Taken together, genetic fate mapping of endogenous cavity macrophages revealed that they did not invade deep into the liver after $\mathrm{CCl}_{4^{-}}, \mathrm{HI}-$, or $\mathrm{CI}$-induced injuries.

Pleural cavity macrophages minimally contribute to lung repair. To further explore whether repair of other visceral organs may involve cavity macrophages, we next studied if pleural cavity macrophages could invade into the lung for its repair and regeneration after injury. We used a lung alveolar-injury model induced by bleomycin, CI or lipopolysaccharide (LPS) to examine the contribution of cavity macrophage in lung repair (Fig. $3 \mathrm{a}$ and Supplementary Fig. 5f). Whole-mount fluorescence images of lungs collected from G6Mø-CreER;R26-tdTomato mice showed a tdTomato $^{+}$region in the lung from the CI mice, and rare sporadic tdTomato ${ }^{+}$cells on the surface of the lungs from Sham, bleomycin- or LPS-treated mice (Fig. 3b and Supplementary Fig. 5g). Sirius Red staining on lung sections showed a significant increase of tissue fibrosis in the bleomycin, CI and LPS groups, compared with the Sham control (Fig. 3c and Supplementary Fig. 5h). Of note, the surface of the lung in the CI group exhibited thickened mesothelial cell layers enriched for fibrosis (Fig. 3c). Next, we performed immunostaining of lung sections for tdTomato, F4/80 and GATA6, and we found markedly greater staining for $\mathrm{F} 4 / 80^{+}$macrophages in the bleomycin- and LPS-treated mice compared with that of the Sham control (Fig. 3d and Supplementary Fig. 5i). Notably, the GATA6 ${ }^{+}$cells detected in the lung parenchyma were not macrophages, as they did not express F4/80 (Fig. 3d). In the lungs of CI-treated mice, tdTomato ${ }^{+}$cavity macrophages were mainly restricted to the thickened mesothelial layers on the surface rather than invasion deep into the lungs (Fig. 3d). We next performed FACS analysis of $\mathrm{CD} 11 \mathrm{~b}^{+} \mathrm{F} 4 / 80^{+}$ cells collected from injured regions of the lungs from the CI, bleomycin- and LPS-treated mice, and found that a negligible percentage of macrophages $(\sim 0.03 \%)$ expressed tdTomato (Fig. 3e,f and Supplementary Fig. 5j). These data demonstrate that 
a

Experimental design

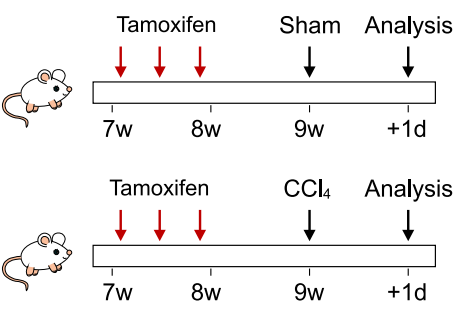

Cwo $\begin{array}{ccc}\text { Tamoxifen } & \text { HI } & \text { Analysis } \\ \frac{1}{7 \mathrm{w}} \quad \text { 8w } & 9 \mathrm{w} & +1 \mathrm{~d}\end{array}$

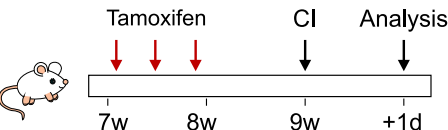

b

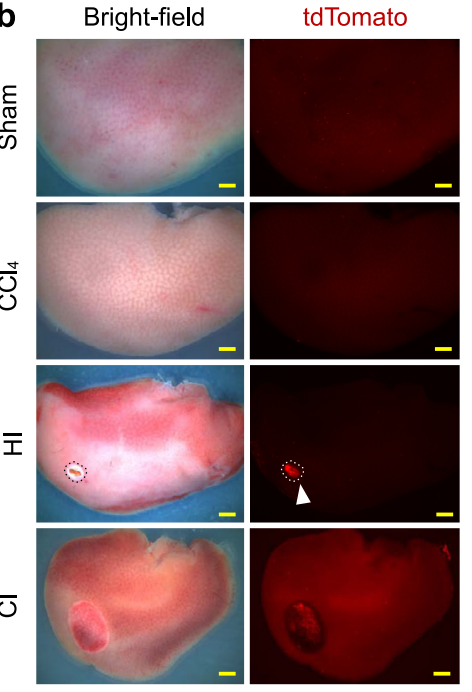

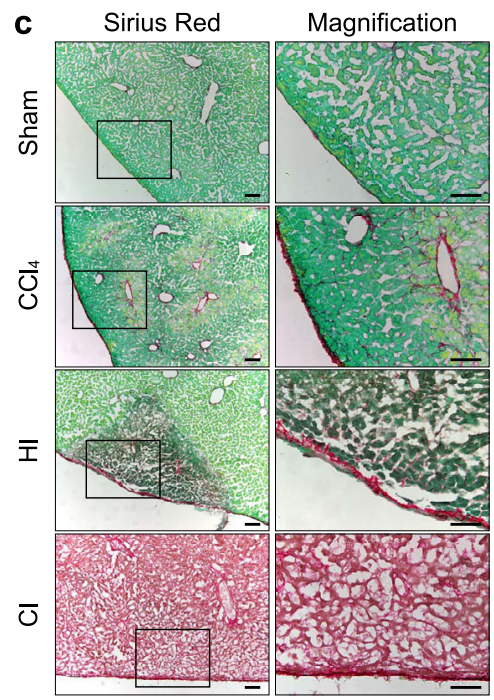

d
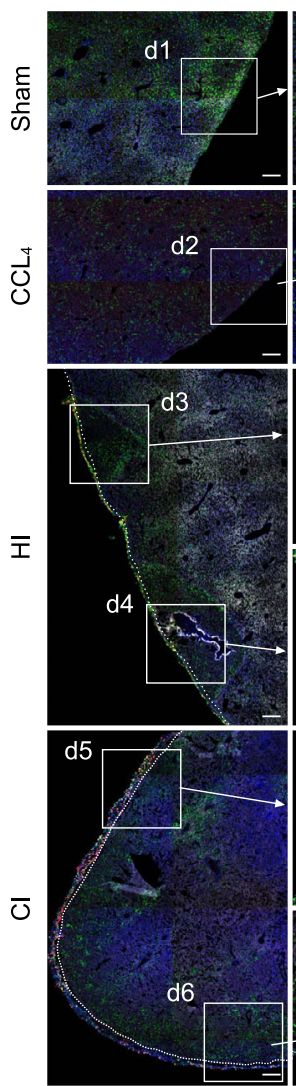
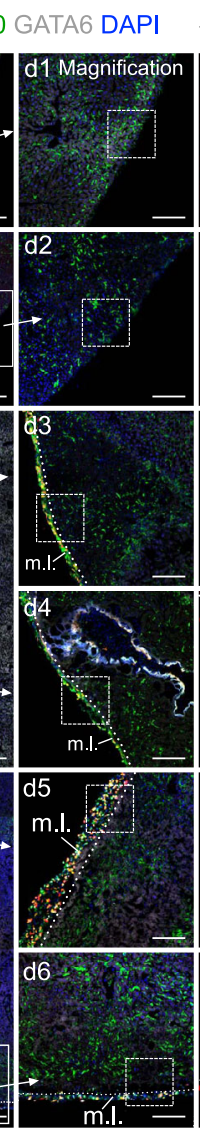
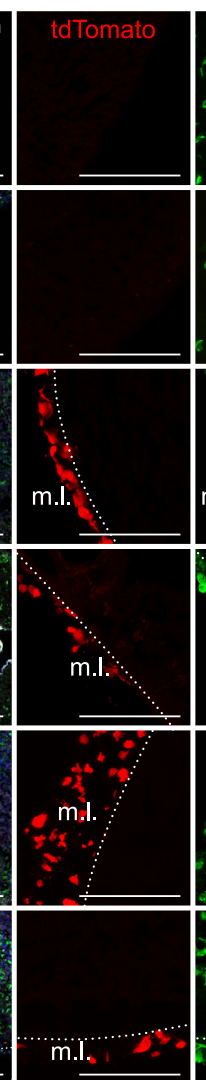

split channel
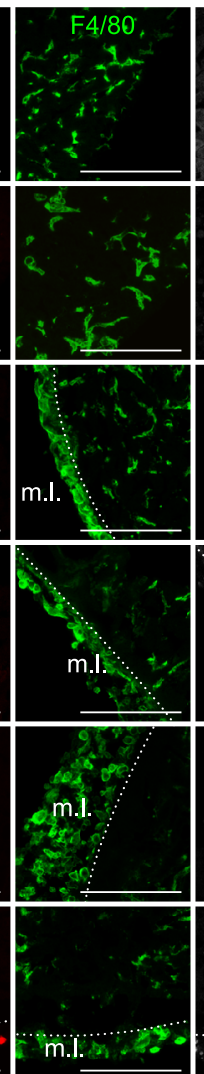
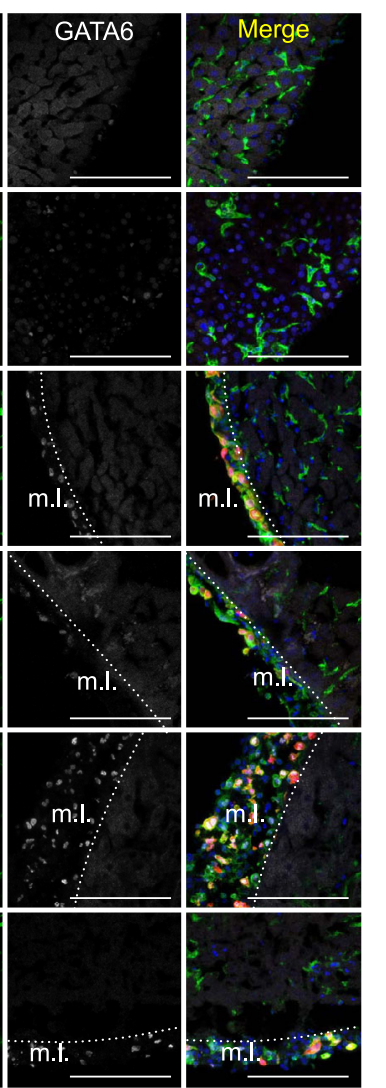

e

Gated on $\mathrm{CD} 11 \mathrm{~b}^{+} \mathrm{F} 4 / 80^{+}$

¿
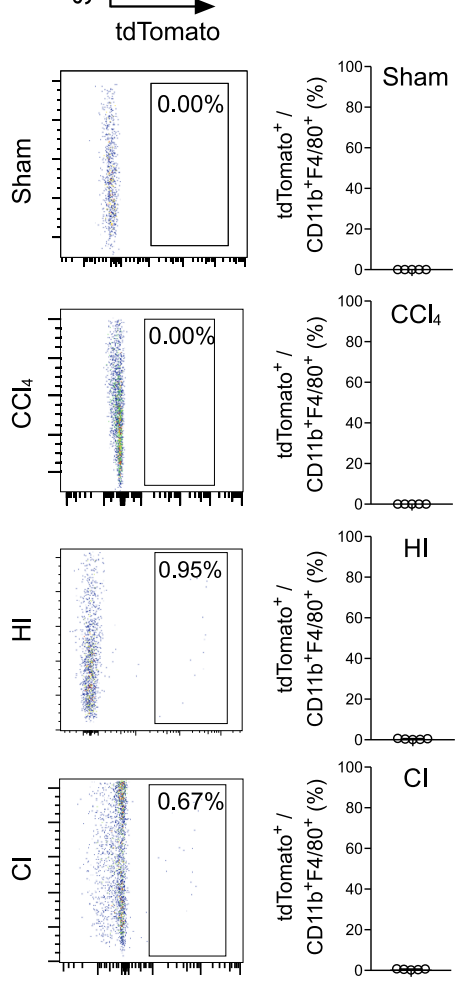

Fig. $\mathbf{2}$ Peritoneal macrophages do not invade deep into the liver parenchyma after $\mathbf{C C l}_{\mathbf{4}}, \mathbf{H I}$, or $\mathbf{C l}$ treatment. a Schematic figure showing experimental strategy. $\mathrm{HI}$ heat injury, $\mathrm{Cl}$ cryoinjury. b Whole-mount bright-field and fluorescent images of livers with different injury models. Arrowheads, injury site. Scale bars, $1 \mathrm{~mm}$. c Sirius red staining of liver tissue sections from livers treated with $\mathrm{CCl}_{4}, \mathrm{Cl}, \mathrm{HI}$, or sham operation. Boxed regions are magnified. Scale bars, $100 \mu \mathrm{m}$. d Immunostaining for tdTomato, GATA6, and F4/80 on the injured region of the liver section. m.l., mesothelial layer. Boxed regions are magnified. Scale bars, $100 \mu \mathrm{m}$. e FACS analysis and quantification of the percentage of F4/80+ macrophages expression tdTomato. Data are the mean \pm $\mathrm{SD} ; n=5$ mice per group. Each image is representative of five individual biological samples (b-d). Source data are provided as a Source Data file. 
a

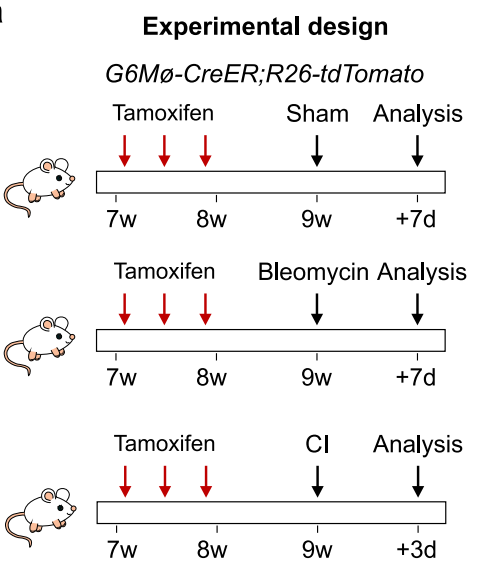
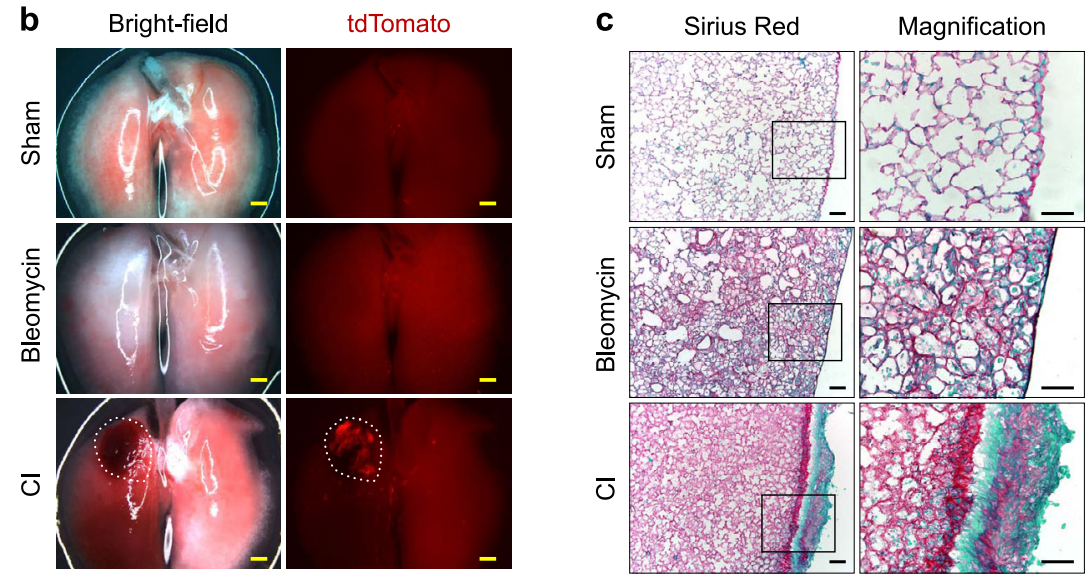

d
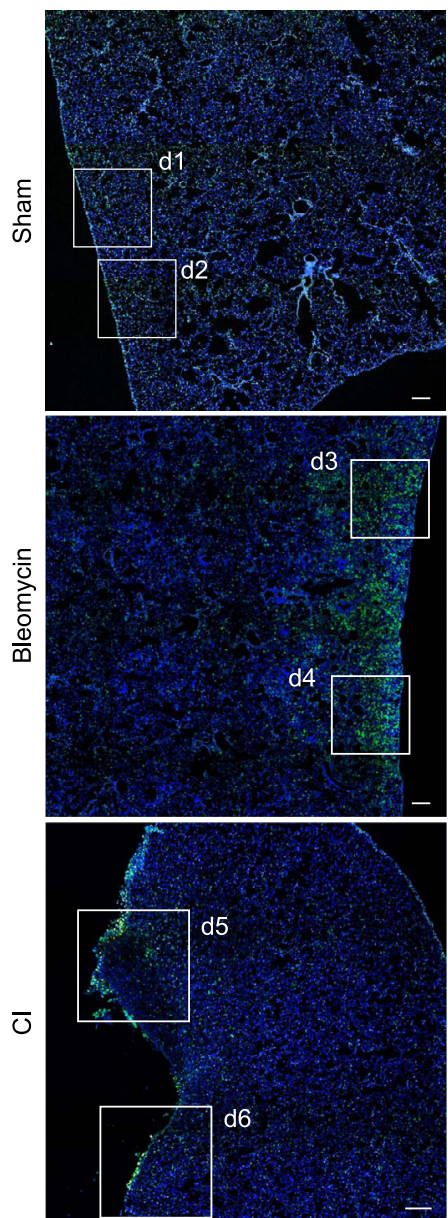
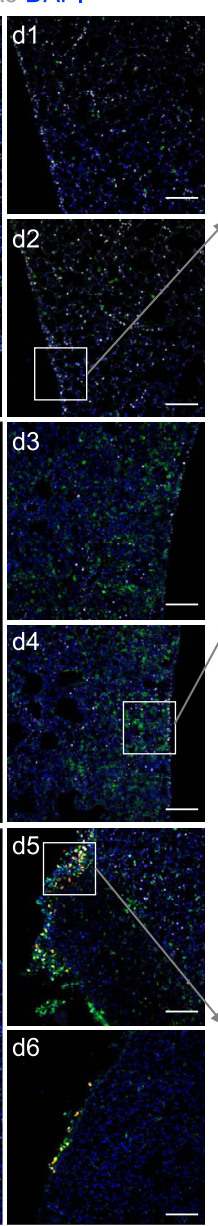
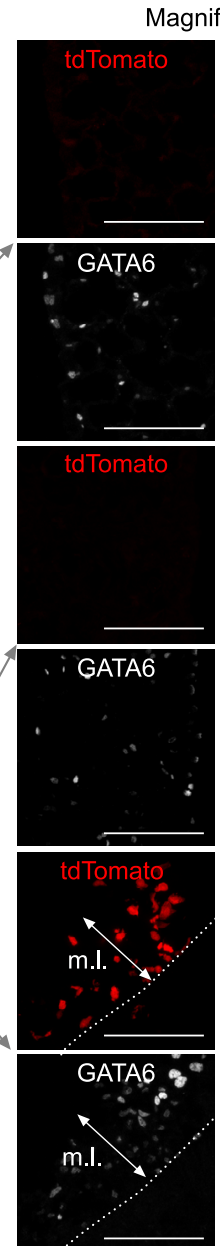

gnification
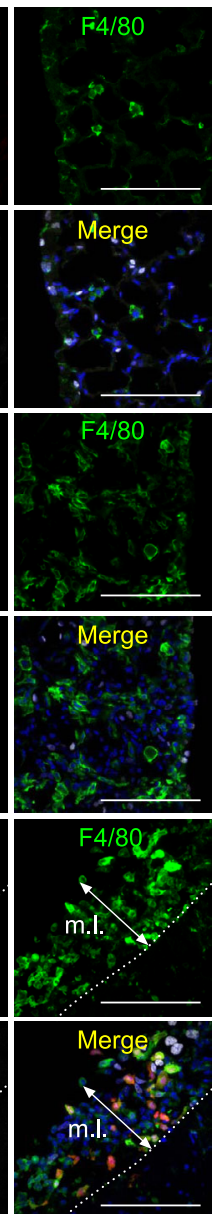

e
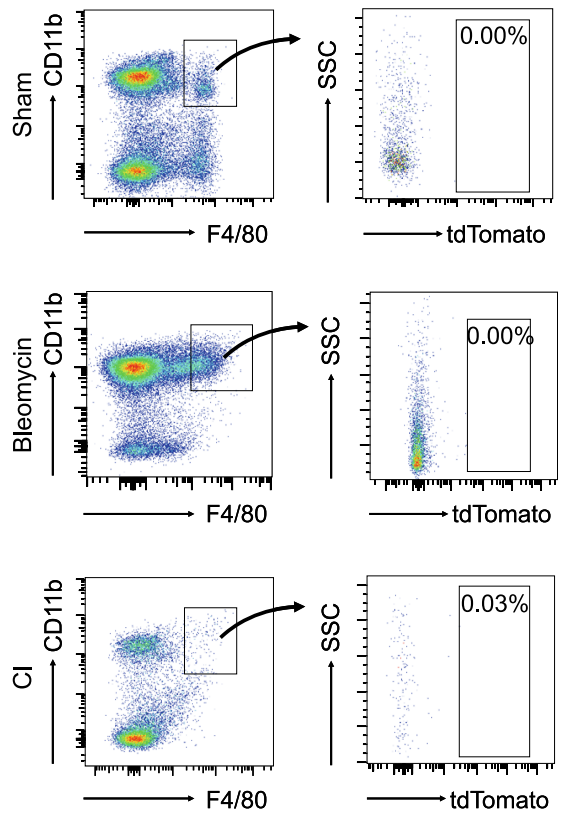

f
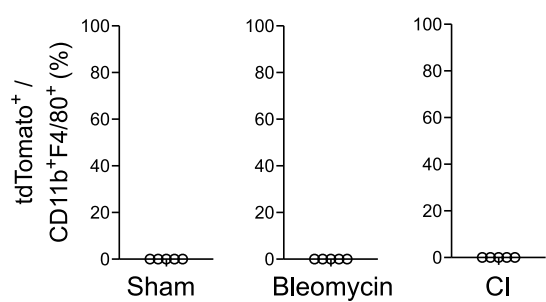

Fig. 3 Pleural macrophages do not invade deep into the lung parenchyma after bleomycin treatment or Cl. a Schematic figure showing experimental strategies. Cl cryoinjury. b Whole-mount bright-field and fluorescent images of lungs after different injuries. Dotted line, $\mathrm{Cl}$ region. Scale bars, 1 mm. c Sirius red staining of lung tissue sections after bleomycin or cryoinjury in lungs. Boxed regions are magnified. Scale bars, $100 \mu \mathrm{m}$. $\mathbf{d}$ Immunostaining for tdTomato, GATA6, and F4/80 on injured regions of lungs. m.l., mesothelial layer. Boxed regions are magnified. Scale bars, $100 \mu \mathrm{m}$. e, f FACS and quantification analysis of the percentage of macrophages expressing tdTomato from injury regions of the lung. Data are the mean \pm SD; $n=5$ mice per group. Each image is representative of five individual biological samples (b-d). Source data are provided as a Source Data file.

pleural cavity macrophages minimally invade the lung, suggesting that they do not contribute to lung repair and regeneration.

Ablation of cavity macrophages do not significantly affect visceral organ repair. It has been previously reported that in a
$\mathrm{CCl}_{4}$ model of organ fibrosis that clodronate liposome-mediated depletion of cavity macrophages results in a greater loss of body weight compared with control mice ${ }^{16}$. Whether clodronate had any side effect on liver hepatocytes or other macrophage populations during recovery from injury remained unclear. To 
a

Experimental design

G6Mø-CreER;R26-tdTomato

G6Mø-CreER;R26-tdTomato/iDTR

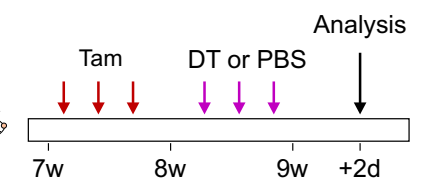

b

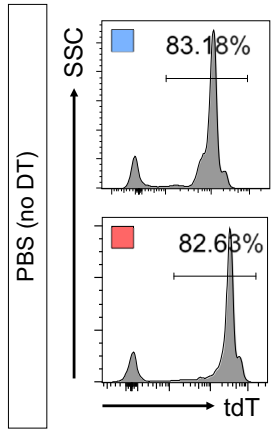

d

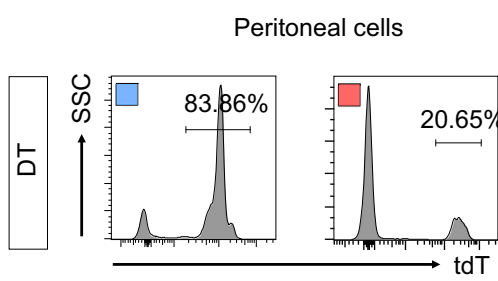

C

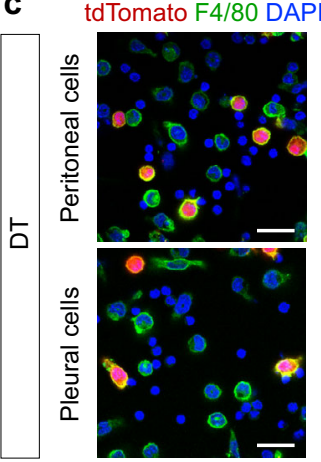

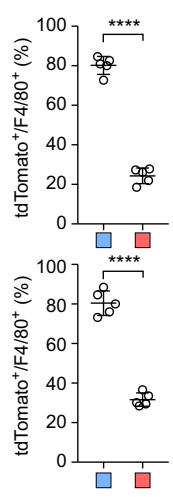

Pleural cells

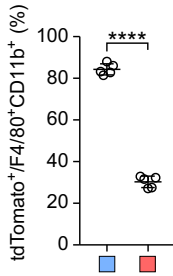

e

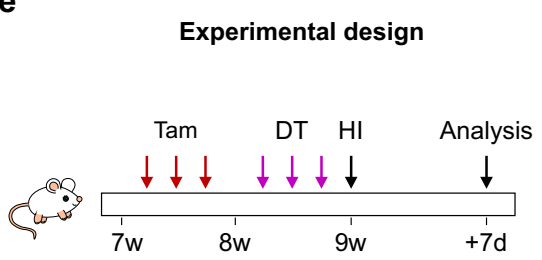

Experimental design
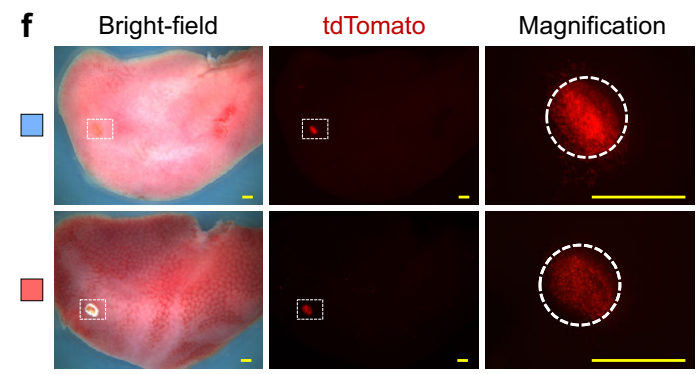

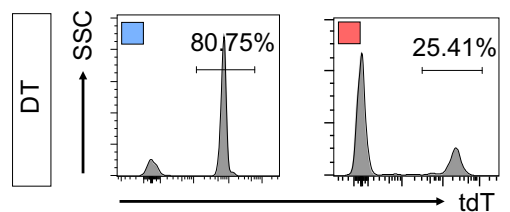

h

\section{Experimental design}

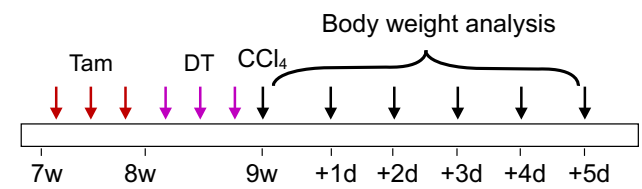

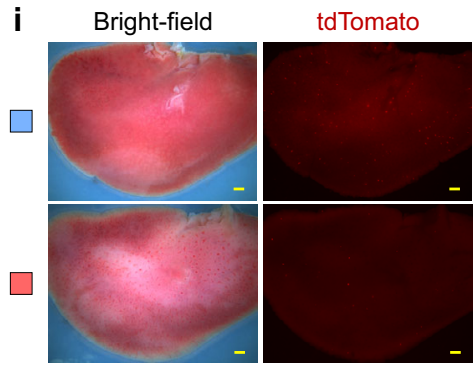

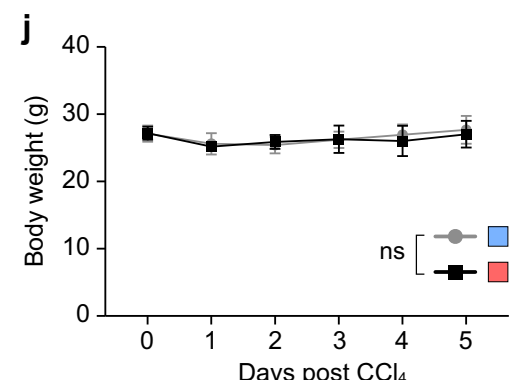

Fig. 4 Genetic ablation of cavity macrophages did not affect liver repair after injury. a Schematic figure showing the experimental design. DT diphtheria toxin. $\mathbf{b}$ Flow cytometric and quantification analysis of the percentage of CD11b+F4/80+ macrophages expressing tdTomato from peritoneal cavity after PBS treatment (no DT). Data are the mean \pm SD; $n=5$ mice per group; ns nonsignificant. c Immunostaining for tdTomato and F4/80 on dissociated cells from the peritoneal or pleural cavity after DT treatment of the G6Mø-CreER;R26-tdTomato/iDTR mice. Quantification of the percentage of F4/80+ macrophages expressing tdTomato. Data are the mean \pm SD; $n=5$ mice per group; ${ }^{\star \star \star \star} P<0.0001$. Each image is representative of five individual biological samples. $\mathbf{d}$ Flow cytometric and quantification analysis of the percentage of CD11b+F4/80+ macrophages expressing tdTomato in the pleural cavity, and peritoneal cavity, respectively. Data are the mean \pm SD; $n=5$ mice per group; ${ }^{\star \star \star \star} P<0.0001$. e Schematic figure showing the experimental design. HI heat injury. $\mathbf{f}$ Whole-mount bright-field and fluorescence images of livers at 7 days after $\mathrm{HI}$. Boxed regions are magnified; circles, heat injury site. Each image is representative of eight individual biological samples. $\mathbf{g}$ Quantification of injury size from $4 \mathrm{~h}$ to 7 days after heart injury. Data are the mean \pm SD; $n=8$ mice per group; ns nonsignificant. $\mathbf{h}$ Schematic figure showing the experimental design. $\mathbf{i}$ Whole-mount bright-field and fluorescence images of livers at 5 days after $\mathrm{CCl}_{4}$ treatment. Each image is representative of five individual biological samples. $\mathbf{j}$, Quantification of body weight at different days after $\mathrm{CCl}_{4}$ treatment. Data are the mean $\pm \mathrm{SD} ; \mathrm{n}=5$ mice per group; $\mathrm{ns}$, non-significant. Scale bars: yellow, $1 \mathrm{~mm}$; white, $100 \mu \mathrm{m}$. $P$ value was calculated by unpaired two-sided Student's $t$ test $(\mathbf{b}-\mathbf{d}, \mathbf{g})$ or two-way ANOVA coupled with multiple comparisons $(\mathbf{j})$. Source data are provided as a Source Data file.

specifically dissect the reparative function of $\mathrm{GATA}^{+}$cavity macrophages after liver injury, we crossed G6Mø-CreER;R26tdTomato with the inducible diphtheria toxin receptor (DTR) mouse line R26-iDTR ${ }^{35}$ to allow selective ablation of GATA6 ${ }^{+}$ cavity macrophages by subsequent DT treatment ${ }^{36,37}$ (Fig. 4a). Phosphate-buffered saline (PBS) injection in the G6Mø-CreER;
R26-tdTomato/iDTR mice did not result in any significant loss of labeled cavity macrophages (Fig. 4b). When we are immunostained for tdTomato and F4/80 to identify cavity macrophages, we found significantly lower numbers of tdTomato ${ }^{+}$macrophages from the G6Mø-CreER;R26-tdTomato/iDTR mice compared to the G6Mø-CreER;R26-tdTomato mice after DT 
treatment (Fig. 4c). Further, when we performed FACS analysis, we confirmed a significantly lower percentage of tdTomato ${ }^{+}$ macrophages among peritoneal or pleural macrophages (Fig. $4 \mathrm{~d}$ ), indicating successful genetic ablation of the majority of tdTomato + macrophages. After Tam and DT treatments, we then induced $\mathrm{HI}$ and collected liver samples at $4 \mathrm{~h}$ and 7 days after injury (Fig. 4e). There were no significant differences in the injury size between G6Mø-CreER;R26-tdTomato/iDTR mice and G6MøCreER;R26-tdTomato mice after DT treatment (Fig. 4f, g). Furthermore, in the $\mathrm{CCl}_{4}$ model, in which we examined body weight every day for 5 days after treatment (Fig. 4h), we did not detect any morphological changes in the liver (Fig. 4i) nor differences in body weight (Fig. 4j) between G6Mø-CreER;R26-tdTomato/iDTR mice and G6Mø-CreER;R26-tdTomato mice. To further confirm the impact of cavity macrophages in $\mathrm{CCl}_{4}$-induced liver injury, we measured the levels of alanine aminotransferase (ALT) and aspartate aminotransferase (AST) in serum at a different time point after $\mathrm{CCl}_{4}$ treatment (Supplementary Fig. 9a-c). Genetic ablation of cavity macrophages did not significantly impact the ALT or AST level, or severity of tissue fibrosis, compared with controls treated with $\mathrm{CCl}_{4}$ (Supplementary Fig. 9d-g). The above results suggested that ablation of cavity macrophages did not significantly affect visceral organ repair after injury.

Knockout of Gata6 in cavity macrophages has no impact on visceral organ repair. GATA6 has been reported to play an essential role in cavity macrophage-specific function, phenotype, and proliferation ${ }^{20}$. To address whether impaired cavity macrophage function would markedly influence tissue repair, we crossed CD45-Dre;Gata6-iCreER;R26-tdTomato mice with those harboring a Gata6-flox allele and generated CD45-Dre;Gata6iCreER/flox;R26-tdTomato mice. We then treated the newly generated mice with Tam three times between weeks 7 and 8 of age to delete Gata6 and to genetically label cavity macrophages, followed by FACS analysis 1 week later (Fig. 5a). Upon FACS analysis, we observed a significantly lower percentage of tdTomato $^{+}$macrophages in both the peritoneal and pleural cavities of CD45-Dre;Gata6-iCreER/flox;R26-tdTomato mice compared with those in CD45-Dre;Gata6-iCreER;R26-tdTomato control mice (Fig. 5b). We next followed the TAM-treated mice for another four weeks after the initial analysis (Fig. 5c). We observed a further reduction of tdTomato ${ }^{+}$cavity macrophages over time (Fig. 5d-f), which is consistent with previous studies showing that Gata6 deficiency results in dysregulated peritoneal macrophage proliferative renewal ${ }^{20,22}$. In a separate cohort of TAM-treated mice we injured the liver via $\mathrm{HI}$ and collected tissues for analysis 1-week later (Fig. 5g). By whole-mount fluorescence imaging of the livers we found no significant difference in injury size between CD45-Dre;Gata6-iCreER/flox;R26-tdTomato mice and CD45-Dre; Gata6-iCreER;R26-tdTomato control mice (Fig. 5h, i). In yet another cohort of TAM-treated mice we also induced injury via $\mathrm{CCl}_{4}$ treatment and examined their body weight every day afterward for 5 days (Fig. 5j). We did not observe any difference in the gross morphology of the liver between the two groups of mice (Fig. 5k). Nor did we find any significant difference in body weight (Fig. 5l). Next, the serum ALT and AST levels, and fibrosis were evaluated at the indicated times after $\mathrm{CCl}_{4}$ stimulation, and we found that no significant difference between CD45-Dre;Gata6iCreER/flox;R26-tdTomato mice and CD45-Dre;Gata6-iCreER; R26-tdTomato control mice (Supplementary Fig. 9). The combined results above indicate that impaired function of cavity macrophages did not have a noticeable effect on the organ repair.

Gata6 heterozygosity does not affect cavity macrophage function. Considering that Gata6 is functionally essential for the maintenance of cavity macrophage number and function ${ }^{20,22}$, the lack of invasion of Gata6 $^{+}$cavity macrophages in our system could be due to the Gata6 heterozygosity, in case one allele of which was unintentionally disrupted by Gata6-iCreER knockin. To examine if this possibility was indeed a confounding factor in explaining our results, we examined the number, phagocytosis, and polarization genes of $\mathrm{Gata6}^{+/-}$cavity macrophages in comparison with wild-type controls (Supplementary Fig. 4a). By immunostaining and FACS analysis, we did not find any difference in the number of GATA6 ${ }^{+}$ macrophages between Gata $^{+/-}$and wild-type mice (Supplementary Fig. $4 \mathrm{~b}, \mathrm{c})$. Further, immunostaining and FACS analysis of fluorescent beads phagocytosed by macrophages showed no significant difference between cavity macrophages collected from Gata6 $^{+/-}$and wild-type mice (Supplementary Fig. 4d, e). Quantitative real-time polymerase chain reaction ( $\mathrm{qRT}-\mathrm{PCR}$ ) analysis of polarization genes for M1-like and M2-like macrophages revealed no significant difference in expression between the Gata6 $^{+/-}$and wild-type Gata6 ${ }^{+/+}$ groups (Supplementary Fig. 4f). The above data indicate that cavity macrophages from heterozygous Gata6 mice have normal function.

Generation of Gata6-iCreER2 for tracing cavity macrophages without Gata6 loss. Finally, we generated a new allele of Gata6$i C r e E R 2$ in which we inserted rox-Stop-rox-P2A-CreER into the $3^{\prime}$ UTR of the Gata6 gene, thus maintaining the normal structure of the coding sequence of the endogenous gene (Fig. 6a). We then crossed mice harboring this new allele of Gata6-iCreER2 with those carrying CD45-Dre for targeting the $\mathrm{CD} 45^{+} \mathrm{Gata}^{+}$cell population (Fig. 6b). We then crossed CD45-Dre;Gata6-iCreER2 (G6Mø-CreER2) mice with R26-tdTomato mice to enable genetic lineage tracing of cavity macrophages after Tam treatment (Fig. $6 c)$. FACS analysis revealed both high efficiency and specificity of the G6Mø-CreER2 allele in the labeling of pleural and peritoneal cavity macrophages (Fig. 6d, e). Immunostaining of isolated cavity cells for tdTomato, F4/80 and GATA6 confirmed that the majority of cavity macrophages were genetically labeled, and almost all labeled cells were macrophages (Fig. 6f, g).

We then used Tam-treated G6Mø-CreER2;R26-tdTomato mice for $\mathrm{CCl}_{4}$-induced injury and examined the recruitment of tdTomato ${ }^{+}$ cavity macrophages into the liver. By whole-mount fluorescence imaging of livers from $\mathrm{CCl}_{4}$-treated mice, we found very few tdTomato $^{+}$cells on the surface of the liver (Fig. 6h). Likewise, by immunostaining liver sections for F4/80, tdTomato, and GATA6 we found no tdTomato ${ }^{+}$macrophages in the parenchyma of injured livers (Fig. 6i). Of note, GATA6 was also detected in the injured liver, indicating its broad expression in non-cavity macrophages of the liver after $\mathrm{CCl}_{4}$ injury (Fig. 6i). Next, immunostaining for Kupffer cells marker CLEC4F on liver sections of $\mathrm{CCl}_{4}$-treated mice indicated that GATA6 $^{+}$tdTomato $^{-}$cells were mainly resident Kupffer cells (Fig. 7a, b). Furthermore, by using Ms4a3-CreER; R26-tdTomato mice, we could trace the monocyte-derived cells under $\mathrm{CCl}_{4}$ administration ${ }^{38}$. Immunostaining for $\mathrm{F} 4 / 80$, tdTomato, and GATA6 on liver sections suggested that most $\mathrm{GATA}^{+}{ }^{+\mathrm{tdT}}$ mato ${ }^{-}$did not come from monocytes (Fig. 7c, d). Immunostaining for $\mathrm{CD} 11 \mathrm{~b}$ and CCR2 on liver sections of G6Mø-CreER2;R26tdTomato mice showed significantly increased expression levels in GATA6 $^{+}$macrophages in injured areas (Fig. 7e-h). These data independently support the notion that cavity macrophages contribute minimally, if at all, to tissue repair and regeneration after pharmacologically induced liver injury.

\section{Discussion}

In this study, we generated a genetic system to target cavity macrophages. By employing dual recombinase-mediated genetic lineage tracing, we specifically and efficiently traced GATA6 ${ }^{+}$ cavity macrophages during tissue homeostasis and injuries. In 
a

\section{Experimental design}

CD45-Dre;Gata6-iCreER;R26-tdTomato

CD45-Dre;Gata6-iCreER/flox;R26-tdTomato

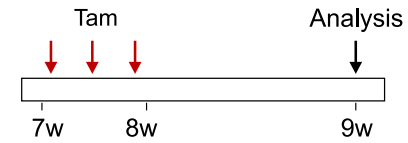

C
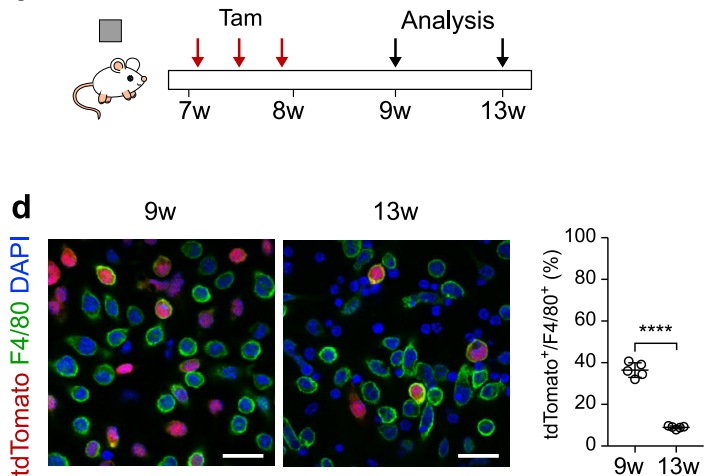

b
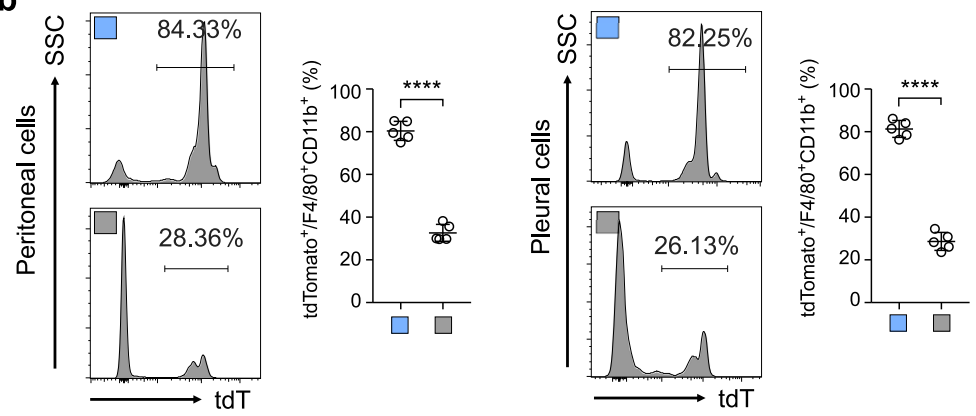

e
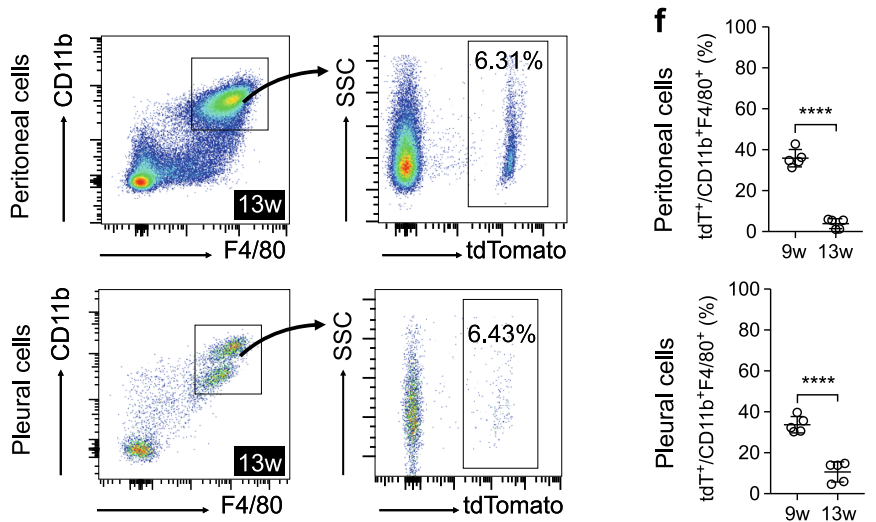

g

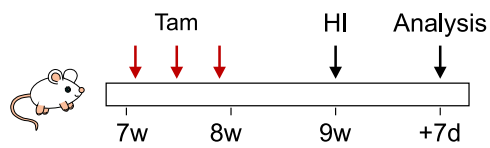

h

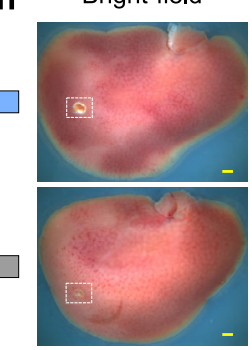

tdTomato

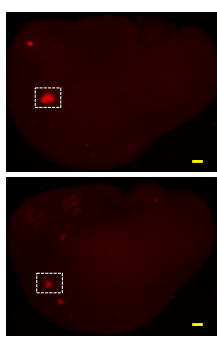

Magnification i
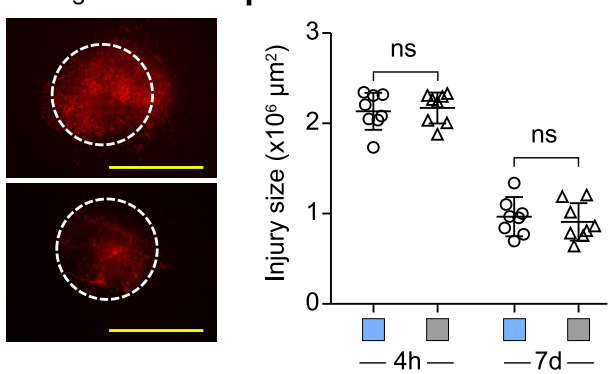

$\mathbf{k}$

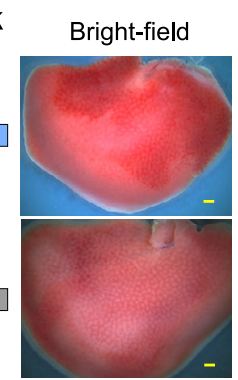

Experimental design
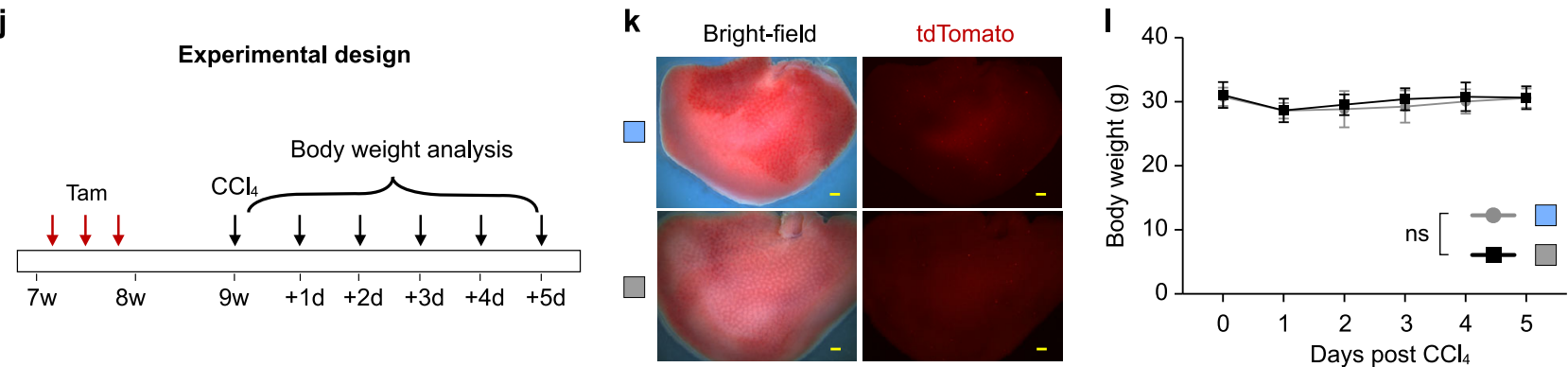

Fig. 5 Gata6 deletion in cavity macrophages did not affect injury repair in the liver. a Schematic figure showing the experimental design. $\mathbf{b}$ Flow cytometric and quantification analysis of the percentage CD11b+F4/80+ macrophages expressing tdTomato from the pleural and peritoneal cavity. Data are the mean \pm SD; $n=5$ mice per group; ${ }^{\star \star \star \star} P<0.0001$. c Schematic figure showing the experimental design. $\mathbf{d}$ Immunostaining for tdTomato and F4/80 on dissociated cells from peritoneal cavity at 9 weeks and 13 weeks after TAM. Data are the mean \pm SD; $n=5$ mice per group. ${ }^{\star \star \star \star} P<0.0001$. Each image

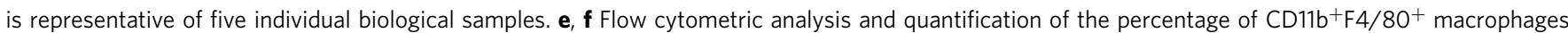
expressing tdTomato. Data are the mean $\pm \mathrm{SD} ; n=5$ mice per group; ${ }^{\star \star \star \star} P<0.0001$. $\mathbf{g}$ Schematic figure showing the experimental design. $\mathrm{HI}$ heat injury. $\mathbf{h}$ Whole-mount bright-field and fluorescence images of livers collected at 7 days after heart injury. Boxed regions are magnified; circles, heat injury site. Each image is representative of eight individual biological samples. i Quantification of injury size at $4 \mathrm{~h}$ and 7 days post HI. Data are the mean \pm SD; $n=8$ mice per group; ns nonsignificant. $\mathbf{j}$ Schematic figure showing the experimental design. $\mathbf{k}$ Whole-mount bright-field and fluorescence images of livers collected at 5 days after injury. Each image is representative of five individual biological samples. I Quantification of body weight at different days after $\mathrm{CCl}_{4}$ treatment. Data are the mean $\pm S D ; n=5$ mice per group; ns, non-significant. Scale bars: yellow, $1 \mathrm{~mm}$; white, $100 \mu \mathrm{m}$. $P$ value was calculated by unpaired two-sided Student's $t$ test $(\mathbf{b}, \mathbf{d}, \mathbf{f}, \mathbf{i})$ or two-way ANOVA coupled with multiple comparisons (I). Source data are provided as a Source Data file. 
a

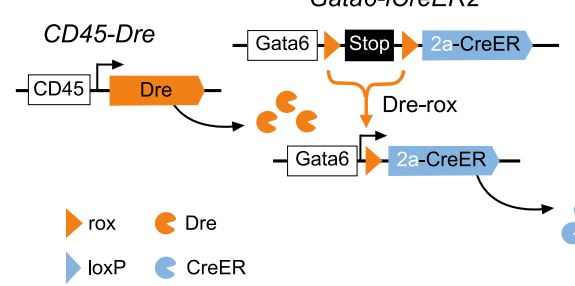

R26-tdTomato

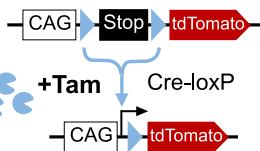

b

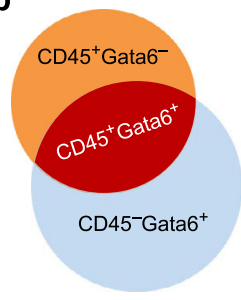

C

CD45-Dre;Gata6-iCreER2 (G6Mø-CreER2) R26-tdTomato

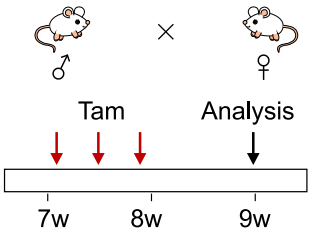

d
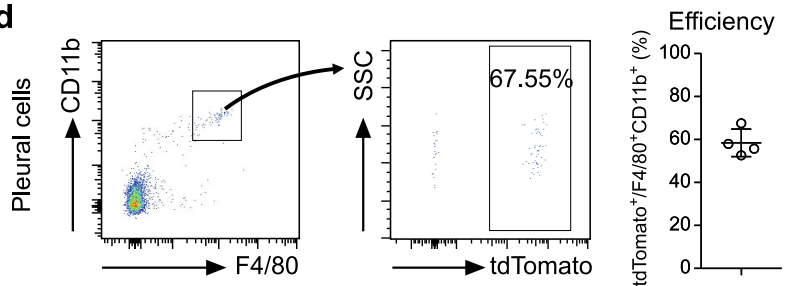

e
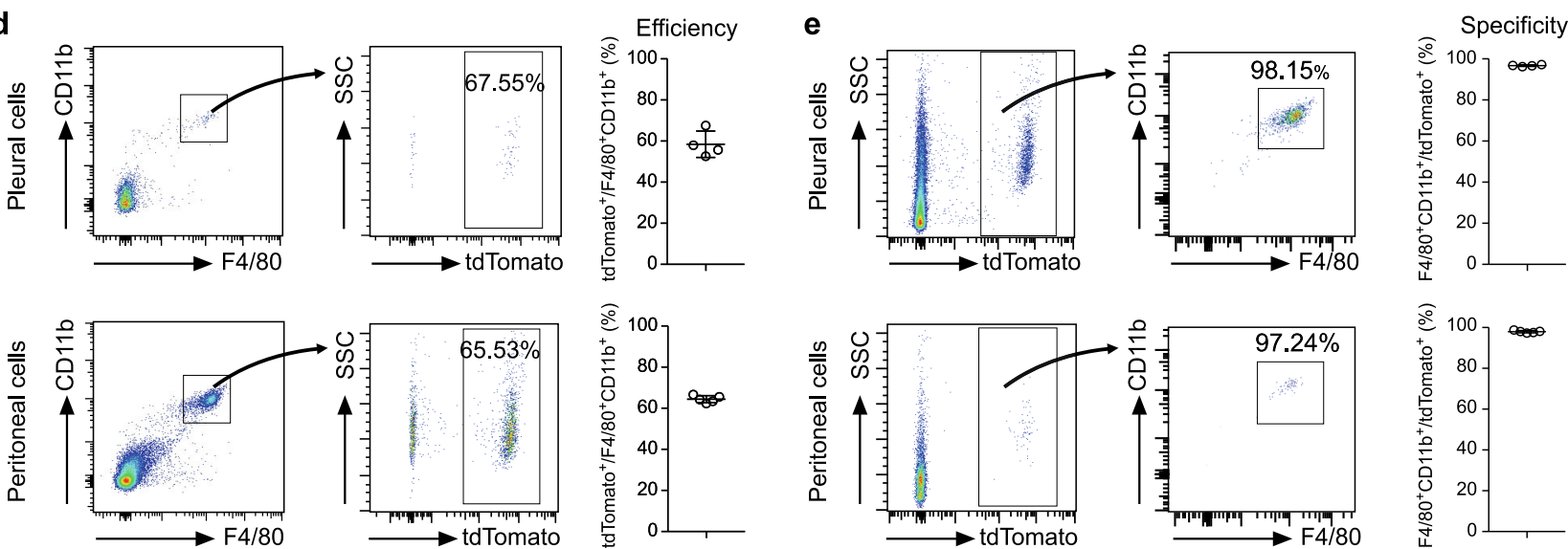

f

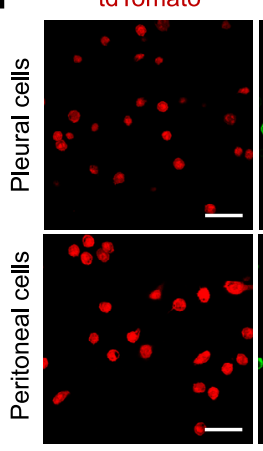

F $4 / 80$

GATA6

Merge + DAPI

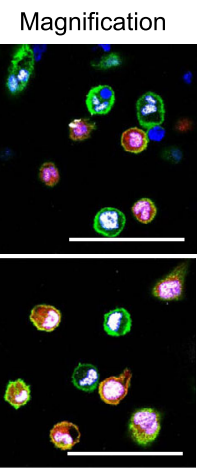

g

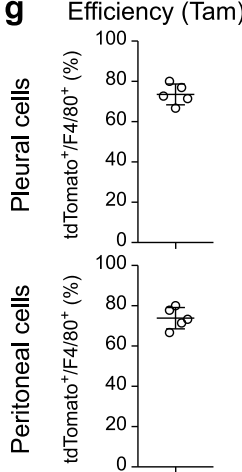

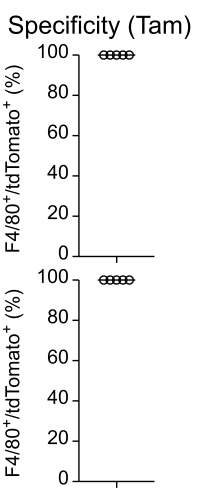
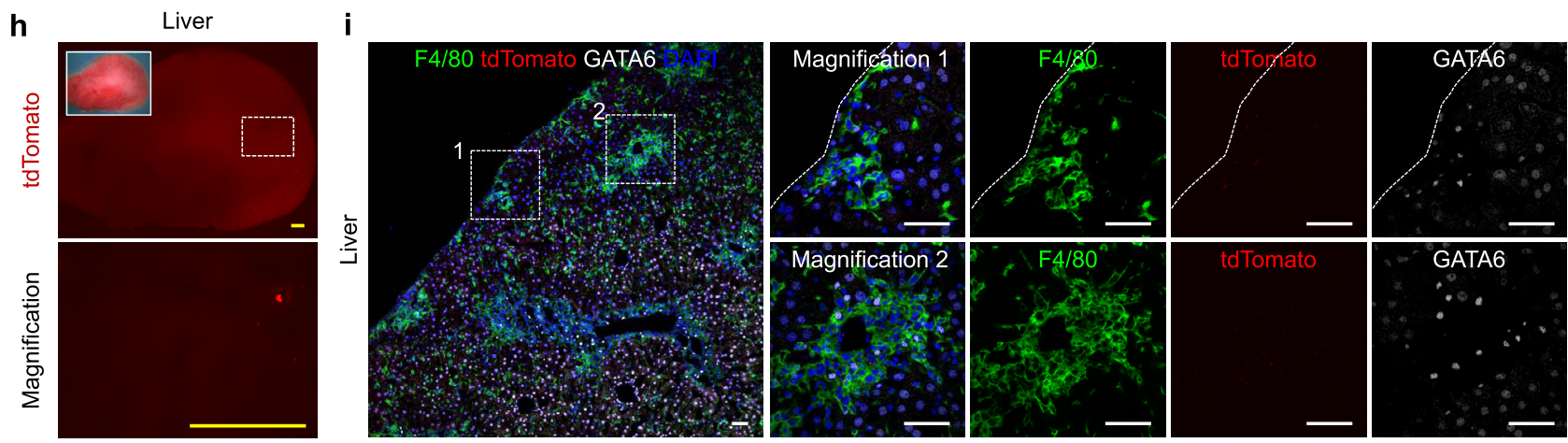

Fig. 6 Generation and characterization of Gata6-iCreER2 for fate mapping of cavity macrophages. a Schematic figure showing experimental strategy. Gata6-iCreER2 uses a knock-in strategy that maintains endogenous Gata6 gene expression. b Intersectional genetics marks CD45+GATA6 ${ }^{+}$cells as tdTomato. c Schematic figure showing experimental design using a second cavity macrophage CreER line (G6Mø-CreER2). d, e Flow cytometric analysis of the percentage of tdTomato ${ }^{+}$cells in macrophages from the pleural and peritoneal cavity (left panel); and the percentage of $\mathrm{F}_{4 / 80^{+}}$macrophages in tdTomato ${ }^{+}$cells from the pleural and peritoneal cavity (right panel). For pleural cells, data are the mean \pm SD; $n=4$ mice per group. For peritoneal cells, data are the mean $\pm \mathrm{SD} ; n=5$ mice per group. $\mathbf{f}$ Immunostaining for tdTomato, GATA6, and F4/80 on dissociated cells from the pleural or peritoneal cavity. Boxed regions are magnified. $\mathbf{g}$ Quantification of the percentage of $\mathrm{F} 4 / 80^{+}$macrophages expressing tdTomato, or the percentage of tdTomato ${ }^{+}$ cells expressing F4/80. Data are the mean $\pm \mathrm{SD} ; n=5$ mice per group. $\mathbf{h}$ Whole-mount epifluorescence images of the liver after $\mathrm{CCl}_{4}$ injury. The boxed region is magnified. $\mathbf{i}$ Immunostaining for tdTomato, GATA6, and F4/80 on the injured region of livers. Dotted lines indicate the surface of tissue sections. Boxed regions are magnified. Each image is representative of five individual biological samples $(\mathbf{f}, \mathbf{h}, \mathbf{i})$. Scale bars, yellow, $1 \mathrm{~mm}$; white, $100 \mu \mathrm{m}$. Source data are provided as a Source Data file. 
a

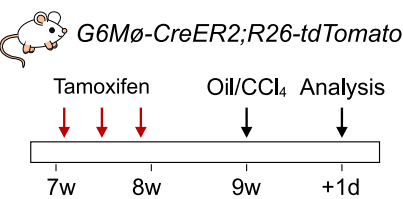

C

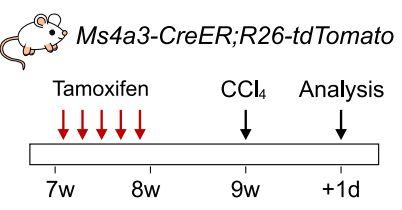

e

g
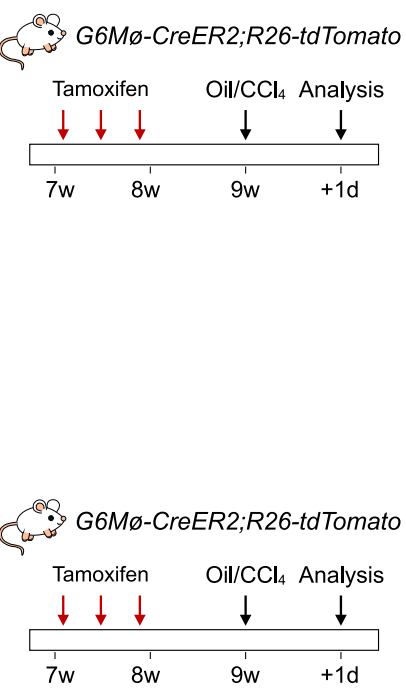

b
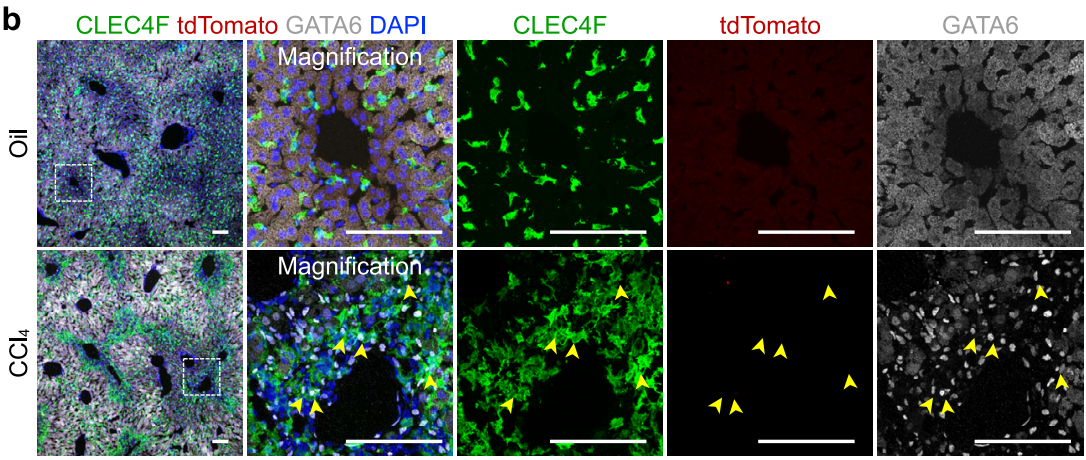

d

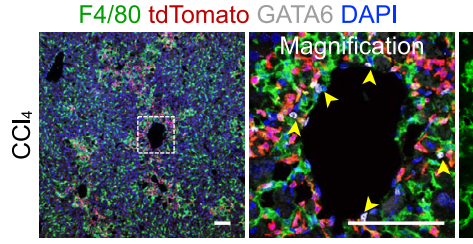

$\mathrm{F} 4 / 80$
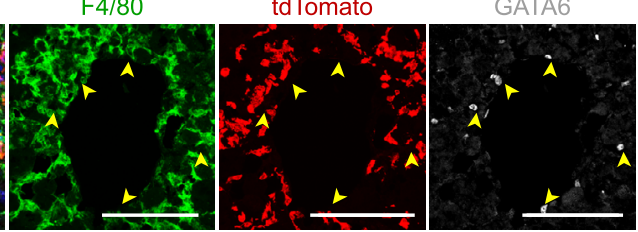

$\mathbf{f}$
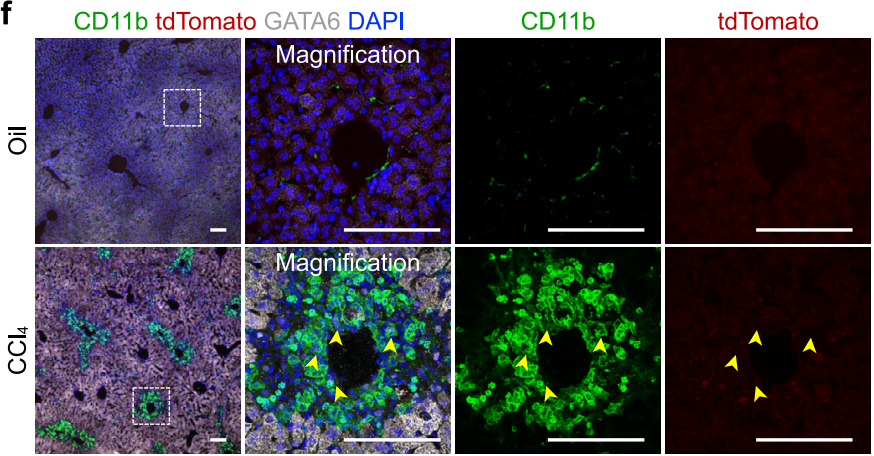

GATA6

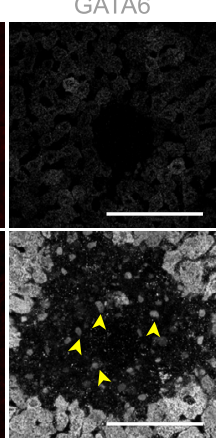

h
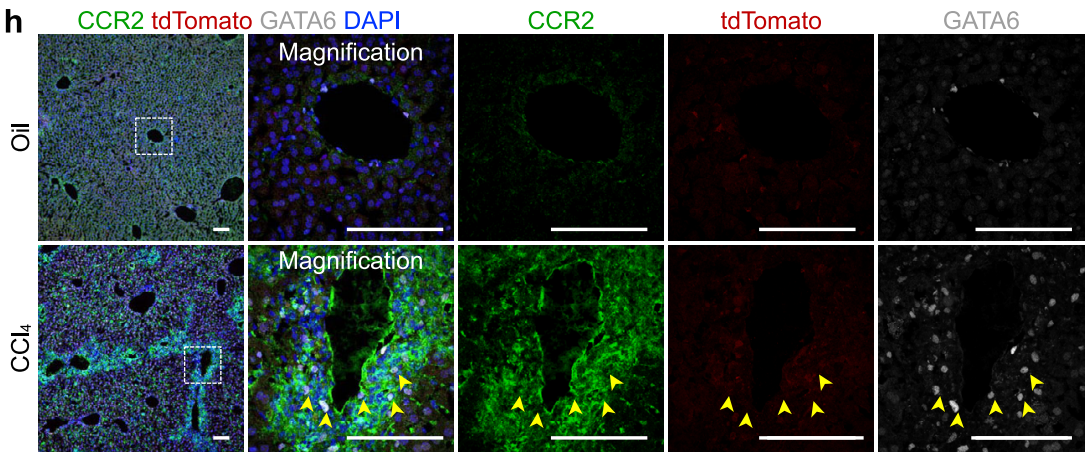

Fig. 7 GATA6 ${ }^{+}$tdTomato ${ }^{-}$cells in the $\mathbf{C C l}_{\mathbf{4}}$-treated livers are mainly resident Kupffer cells. a Schematic figure showing experimental strategy. $\mathbf{b}$ Immunostaining for tdTomato, GATA6, and CLEC4F on liver sections. c Schematic figure showing experimental strategy. d Immunostaining for tdTomato, GATA6, and F4/80 on the injured region of the liver section. e Schematic figure showing experimental strategy. $\mathbf{f}$ Immunostaining for tdTomato, GATA6, and CD11b on liver sections. $\mathbf{g}$ Schematic figure showing experimental strategy. $\mathbf{h}$ Immunostaining for tdTomato, GATA6, and CCR2 on liver sections. Scale bars, $100 \mu \mathrm{m}$. For $\mathbf{b}, \mathbf{d}, \mathbf{f}, \mathbf{h}$ arrowheads indicate GATA6 ${ }^{+}$cells after injury. Boxed regions are magnified. Each image is representative of five individual samples.

contrast to previous reports showing deep invasion and a significant contribution of these cells to the functional recovery of visceral organs after injury 16,17 , our genetic fate mapping approaches reveal that cavity macrophages minimally invaded visceral organs after injury and contributed negligibly to the repair and regeneration of the liver or lung (Fig. 8). Genetic-based cell ablation and Gata6 gene deletion in cavity macrophages did not significantly affect the repair of injured organs. Our study raises concerns about the conclusions of previously reported results based on Gata6 gene activity and cell transplantation for cavity macrophage study ${ }^{16,17}$. Whether cavity macrophages have additional roles in visceral organ repair other than direct invasion remains to be explored further in the future.

Currently, there are no infallible means of tracking cell fate in any organ system. However, genetic fate mapping approaches provide the strongest level of spatiotemporal resolution, hence the level of scientific evidence for in vivo cell origin and fate in multiple field ${ }^{39-44}$. The strength of genetic lineage tracing technology is that it allows for the permanent and irreversible marking of cells and all their descendants in vivo, no matter where they migrate and how 

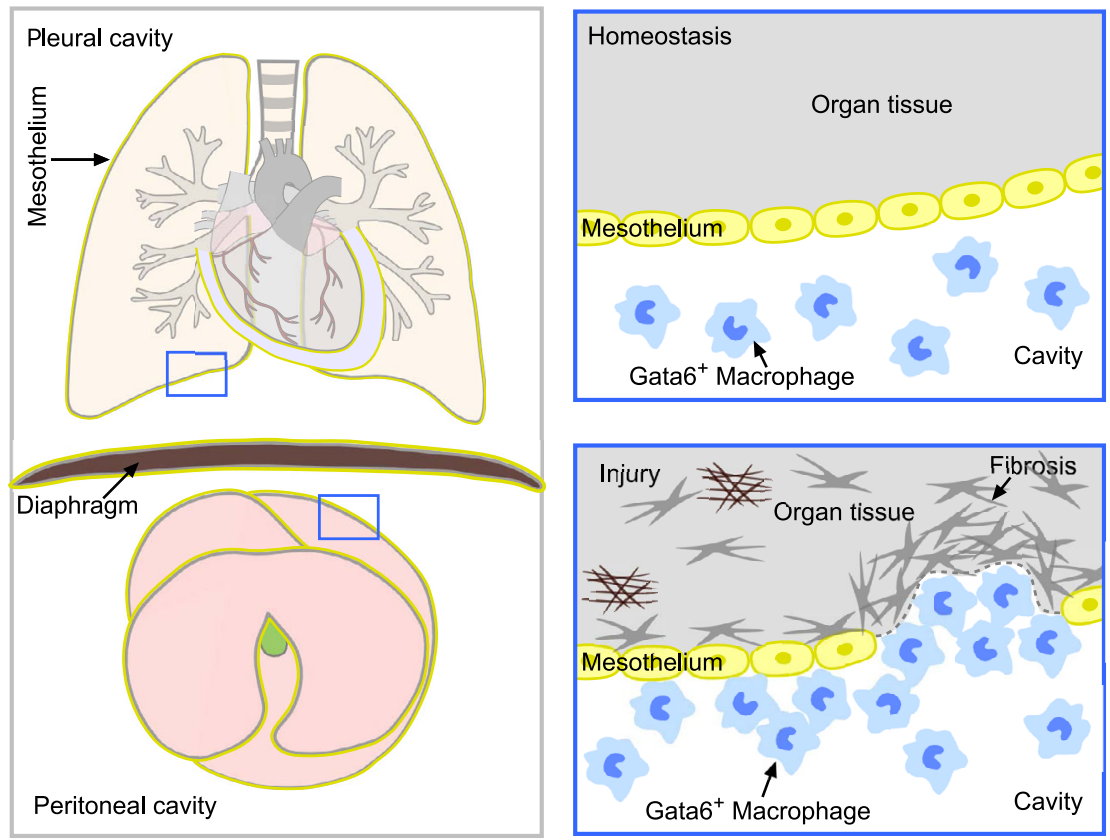

Fig. 8 Pleural and peritoneal cavity macrophages minimally invade visceral organs after injuries. Gata6 ${ }^{+}$cavity macrophages are located in peritoneal and pleural cavities, which are separated by mesothelium that wraps visceral organs such as lung, and liver. After injuries, cavity macrophages are recruited to the surface of visceral organs, but they do not infiltrate into the parenchyma of organs. Nor do they play a functional role in tissue repair and regeneration.

they might evolve during tissue homeostasis or injuries. Further, such a genetic approach allows for the tracing of cells under more physiological conditions that avoid the stressful conditions induced by cell transplantation and in vitro cell manipulation. We, therefore, chose to use a genetic lineage tracing approach to assess cell fate of cavity macrophages in vivo, which provides an orthogonal approach significantly different from the methods used in previous studies $^{16,17}$, which relied mainly on measuring gene activity of cavity macrophage markers and cell transplantation for tracing these cells in visceral organs ${ }^{16}$. In particular, F4/80 staining was used for determining the location of cavity macrophages, which has the caveat of not knowing the origin(s) of these cells ${ }^{16}$. The expression map, e.g., GATA6 expression, is not reliable as gene activity can change depending on the surrounding conditions, due to diverse origins of macrophages, macrophage plasticity, and their dynamic microenvironment ${ }^{1,45-47}$. The cell transplantation experiments, such as the transfer of peritoneal cells from LysM-eGFP mice ${ }^{16}$ or Gata6Venus bone marrow cells transplanted into irradiated mice ${ }^{17}$, are important to define the contribution of cavity macrophages in visceral organs. However, a general caveat to the cell transplantation approach is that the process may represent an artificial state or extraordinary condition that forces these transplanted cells to adopt potentially enhanced functions for which they usually do not display under physiological conditions. Numerous studies have documented the discrepancy of conclusions regarding the interpretation of cell fate based on a cell transplantation approach vs. and one based on genetic fate mapping. For example, hair follicle bulge stem cells give rise to all epidermal lineages upon cell transplantation but only to hair follicle regeneration under physiological conditions ${ }^{48}$. As another example, in contrast to the cell plantation study showing that life-long blood cell production is driven by a small number of multipotent hematopoietic stem cells ${ }^{49}$, a subsequent in situ genetic tracing study revealed that steady-state blood production is actually maintained by the successive recruitment of thousands of clones ${ }^{50}$. In addition, while single multipotent stem cells have the capacity to produce sufficient differentiated progeny to constitute an entire functional mammary gland after transplantation ${ }^{51}$, genetic lineage tracing suggests that unipotent rather than multipotent stem cells contribute to the maintenance of luminal and basal mammary epithelial cells in the adult ${ }^{52}$. Finally, a cell transplantation assay identifies c-Kit ${ }^{+}$multipotent lung stem cells ${ }^{53,54}$, which was subsequently called into question by a genetic lineage tracing study ${ }^{54}$. These compelling examples provide a reasonable explanation as to why the conclusion of our current lineage tracing study may differ from those based on a cell transplantation assay to study the role of cavity macrophages in tissue repair; they also highlight the value of genetic lineage tracing experiments to further interrogate findings from the transplantation study setting.

Taken together, our work here, based on a genetic approach to specifically trace and manipulate cavity macrophages in vivo, suggests that cavity macrophages minimally invade visceral organs after injury to contribute to tissue repair and regeneration, and thus appear to contradict the conclusions of previous studies in this regard $^{16,17}$. Even so, it could be possible that under other circumstances than those tested here cavity macrophages could be activated to invade deep into organs and play an essential role during the repair. But such a paradigm needs to be revisited by other independent groups and by different injury models in the future.

\section{Methods}

Mice. All animal protocols used in this study were approved by the experimental animal facility which has been accredited by the Institutional Animal Care and Use Committee (IACUC) of the State Key Laboratory of Cell Biology, Shanghai Institute of Biochemistry and Cell Biology, Center for Excellence in Molecular Cell Science, University of Chinese Academy of Sciences, Chinese Academy of Sciences. The previously described mouse strains used in this study included R26-iDTR, CAG-Dre, R26-tdTomato, R26-rox-tdTomato, Gataf flox and Ms4a3-CreER mice ${ }^{35,38,55-57}$. The R26-iDTR and Gatafflox mice were originally obtained from The Jackson Laboratory and the stock\# were jax007900 and jax008196, respectively. The CD45-Dre knock-in mouse line was generated by homologous recombination using CRISPR/Cas9. The cDNA encoding Dre recombinase was inserted into CD45 gene locus to replace the endogenous translational start codon and was followed by a transcriptional stop polyA sequence. The Gata6-rox-stop-rox-CreER (Gata6-iCreER) knock-in mouse line was constructed by inserting a rox-stop-roxCreER-WPRE (Woodchuck hepatitis virus regulatory element)-polyA cassette into the Gata6 gene, replacing the endogenous translational start codon. The Gata6$i C r e E R$ strain was generated by conventional homologous recombination using ES 
cells with neomycin for selection. These mouse lines were generated by Shanghai Biomodel Organism Science and Technology Development, Shanghai, China. All mouse lines were maintained in a C57BL/6 genetic background. Mice were housed in accordance with the regulations on mouse welfare and ethics of the Institute of Biochemistry and Cell Biology in groups with 12-h dark-light cycles and had free access to food and water. Both male and female mice of the adult stage beginning at 7 weeks old were included in the study. Tamoxifen (Sigma, T5648) was dissolved in corn oil $(20 \mathrm{mg} / \mathrm{mL})$ and administered by gavage at the indicated time points. For DT administration, mice were injected intraperitoneally with $10 \mathrm{ng} / \mathrm{g}$ body weight DT (dissolved in PBS; Sigma, D0564-1MG). No obvious adverse side effects of DT were detected when administered to the control and R26-iDTR mice. All mice were kept in group housing (2-5 mice per cage) in a specific pathogen-free facility with controlled environmental conditions of temperature $\left(20-25^{\circ} \mathrm{C}\right)$, humidity (30-70\%), and lighting (a 12-h light/dark cycle)

Euthanasia for the animal experiments. For mice, carbon dioxide $\left(\mathrm{CO}_{2}\right)$ was used for euthanasia in an appropriate euthanasia box. After exposure to $\mathrm{CO}_{2}$, a careful assessment was needed to be done to confirm no signs of life in mice, like breathing and heartbeat. Then the mice were removed for the following steps.

Genomic PCR. For genotyping of mice, the biopsies (tail tips) were collected approximately 7 days after birth. These biopsies were digested by tail lysis buffer $(300 \mu \mathrm{l})$ with proteinase $\mathrm{K}(5 \mu \mathrm{l}$, Roche, 3115852001$)$ at $55^{\circ} \mathrm{C}$ for at least $4 \mathrm{~h}$, and $600 \mu \mathrm{l}$ absolute $\mathrm{EtOH}$ was added for centrifugation at $10,656 \mathrm{~g}$ for $2 \mathrm{~min}$. The supernatant was discarded and $600 \mu \mathrm{l} 70 \% \mathrm{EtOH}$ was added for centrifugation at $10,656 \mathrm{~g}$ for $2 \mathrm{~min}$. Afterward, the liquid was discarded and the DNA air-dried. Totally, $200 \mu$ of distilled water was added to dissolve the DNA, for PCR amplification. Primers and protocol for PCR are available upon request.

Tissue whole-mount fluorescence microscopy. Collected visceral organs were washed in PBS and placed on agar in the required direction for whole-mount bright-field and fluorescence images by using a Zeiss stereoscope (AxioZoom V16). To determine the magnification of specific regions, we used the automated $\mathrm{z}$-stack images acquired with a Zeiss stereoscope (AxioZoom V16).

Tissue collection and IF staining and imaging. After killing of the mice, tissues were fixed in 4\% paraformaldehyde (PFA, Sigma, P6148) for no more than $1 \mathrm{~h}$ at $4{ }^{\circ} \mathrm{C}$. After fixation, the tissues were rinsed in PBS and incubated in $30 \%$ sucrose at $4{ }^{\circ} \mathrm{C}$ overnight. Then, the tissues were embedded in OCT (Sakura, 4583) medium in the required direction and stored at $-80^{\circ} \mathrm{C}$. Sections were cut at a thickness of $10 \mu \mathrm{m}$. Before staining, sections need to be put in a fume hood and dry by wind, then washed 3 times in $1 \times$ PBS for 5 min each. Subsequently, the sections were blocked with $5 \%$ donkey serum in PBST and incubated with appropriately diluted primary antibodies at $4{ }^{\circ} \mathrm{C}$ overnight. Afterward, the sections were washed 3 times in $1 \times$ PBS for 5 min each and then incubated with suited secondary antibodies or other dyes at room temperature for $40 \mathrm{~min}$ in dark. After 3 times $1 \times$ PBS washing, the fixed and stained sections were mounted with $50 \%$ glycerol and nail polishing oil on four edges of the glass coverslip for further analysis. Alexa fluorescence secondary antibodies were used to develop signals. To amplify weak signals, horseradish peroxidase-conjugated antibodies were used. Immunostaining images were acquired by Zeiss confocal microscopy system (LSM710) and Olympus Laser scanning confocal microscope (Fluoview 1200). The ImageJ (NIH) and Photoline (21.00) software were used to analyze images. The primary antibodies used for IF staining were as follows: F4/80 (Abcam, ab6640; 1:500), GATA6 (Cell Signaling Technology, D61E4; 1:500), CD11b (ThermoFisher Scientific, 14-0112-82; 1:400), tdTomato (Rockland, 600-401-379; 1:1000), CD45 (eBioscience, 17-0451-82; 1:400), CLEC4F (R\&D, AF2784; 1:500) and CCR2 (R\&D, FAB5538A-100; 1:500). The secondary antibodies were used as follows, Alexa donkey anti-rabbit 488 (Invitrogen, A21206; 1:1000), Alexa donkey anti rabbit 555 (Invitrogen, A31572; 1:1000), Alexa donkey anti-rabbit 647 (Invitrogen, A31573; 1:1000), Alexa donkey anti-rat 488 (Invitrogen, A21208; 1:1000), Alexa donkey anti-rat 647 (Abcam, ab150155; 1:1000), Alexa donkey anti-goat 488 (Invitrogen, A11055; 1:1000), Alexa donkey anti-goat 647 (Invitrogen, A21447; 1:1000), and Impress goat-anti rat (Vector lab, MP-7444; 1:3).

Cell isolation and flow cytometry. For pleural and peritoneal cavity cells: Mice were anesthetized by hypodermic injection with $1 \%$ pentobarbital sodium. Peritoneal cells were isolated by flushing the peritoneal cavity with a single injection of $8 \mathrm{ml}$ sterile cold PBS ${ }^{58}$. For the pleural cavity, $4 \mathrm{ml}$ sterile cold PBS was fine ${ }^{16,17}$. The retracted cell fluid was centrifuged at $500 \mathrm{~g}$ for $5 \mathrm{~min}$ at $4{ }^{\circ} \mathrm{C}$ and washed with PBS before staining.

For blood cells: Mouse blood was collected into heparin-containing PBS solution. After red blood cell lysing, the cells were washed twice with PBS before staining.

For liver: After killing of the mice, liver biopsies of the injured area were harvested into cold HBSS (Invitrogen, 14026126) with 0.05\% collagenase type IV (Worthington, LS004188) after being perfused in vivo via the portal vein with 30 $\mathrm{ml}$ HBSS. Then the liver was minced into small pieces and digested by $10 \mathrm{ml}$ HBSS containing $0.05 \%$ collagenase Type IV and DNase I $(60 \mathrm{U} / \mathrm{ml}$, Worthington, LS002139) at $37^{\circ} \mathrm{C}$, shaking for $30 \mathrm{~min}$. The liver specimen was filtered through a
$70 \mu \mathrm{m}$ cell strainer. Next, the cells were centrifuged at $50 \mathrm{~g}$ for $1 \mathrm{~min}$ at $4{ }^{\circ} \mathrm{C}$ to collect non-parenchymal cell-enriched supernatant. Then the non-parenchymal cells were purified using centrifugation 33\% Percoll (Sigma, P1644) solution containing $10 \mathrm{U} / \mathrm{ml}$ heparin (Sigma, H3149). After spinning at $500 \mathrm{~g}$ for $15 \mathrm{~min}$ at $4{ }^{\circ} \mathrm{C}, 1 \mathrm{ml}$ Red Blood Cell lysis buffer (eBioscience, 00-4333-57) was added for $5 \mathrm{~min}$ at room temperature. To stop the reaction, $9 \mathrm{ml}$ cold PBS was added and centrifuged at the speed of $500 \mathrm{~g}$ for $5 \mathrm{~min}$ at $4{ }^{\circ} \mathrm{C}$ to discard the supernatant. After red blood cell lysing, the cells were washed twice with PBS before staining.

For lung: After killing, the mice were perfused with $10 \mathrm{ml}$ cold PBS through the right ventricle to flush out blood cells in the lung. Then the mice were inflated through the trachea with $2 \mathrm{ml}$ digestion solution (Collagenase IV $2 \mathrm{mg} / \mathrm{ml}$, FBS 5\% and DNase I 60 U/ml in RPMI-1640 Media. FBS, Gibco, 10099141; RPMI-1640, Invitrogen, 22400089). The lungs were removed and minced into small pieces in $10 \mathrm{ml}$ digestion solution for $30 \mathrm{~min}$ at $37^{\circ} \mathrm{C}$ with shaking and frequent agitation. After digestion, the cells were filtered through a $70 \mu \mathrm{m}$ strainer, centrifuged at $500 \mathrm{~g}$ for $15 \mathrm{~min}$ at $4{ }^{\circ} \mathrm{C}$ to discard the supernatant. Next, cells were incubated in $1 \mathrm{ml}$ Red Blood Cell lysis buffer (eBioscience, 00-4333-57) at room temperature for 5 min. Totally, $9 \mathrm{ml}$ PBS was added and centrifuged at the speed of $500 \mathrm{~g}$ for $5 \mathrm{~min}$ at $4^{\circ} \mathrm{C}$ to discard the supernatant. After red blood cell lysing, the cells were washed twice with PBS before staining.

For cavity cells, blood cells, liver, and lung single-cell suspension, the cells were stained with primary antibodies containing CD45 FITC (eBioscience, 11-0451, 1:200), F4/80 PE-Cy7 (Biolegend, 123114, 1:200), and CD11b APC (eBioscience, 17$0112-81,1: 200)$ at $4^{\circ} \mathrm{C}$ for $30 \mathrm{~min}$. Next, the cells were washed and re-suspended by PBS, and then stained with DAPI (Vector Laboratories) at $4{ }^{\circ} \mathrm{C}$ for $5 \mathrm{~min}$ before FACS. The cells were analyzed using Attune NxT Flow Cytometer (Thermo Fisher Scientific). Data were generated using FlowJo (Tree Star). The gating strategies for flow cytometry data analysis are illustrated in Supplementary Fig. 10.

Cell culture and IF staining and imaging. Before cell culture, the sterile coverslips were coated with $1 \%$ Gelatin (Solarbio, G8061) in the 24-well plate in the cell culture hood for at least $1 \mathrm{~h}$. Then discard the excess liquid and dry the coverslips completely in the cell culture hood. The cavity and blood cells were maintained in Dulbecco's Modified Eagle's Medium (DMEM, Invitrogen, 11965092), supplemented with 10\% fetal bovine serum (Gibco, 10099141) at $37{ }^{\circ} \mathrm{C}$ in the presence of $5 \% \mathrm{CO}_{2}$. After incubation at $37^{\circ} \mathrm{C}$ for $4-6 \mathrm{~h}$, removed the cell culture medium and rinsed the cells 3 times with PBS. The following procedures were carried out using standard procedures.

Sirius red staining. Sirius red staining was used to determine collagen deposition and indicate fibrotic areas in injury models. This was performed using a standard protocol as described previously ${ }^{59}$. In detail, the tissue cryosections were washed in $1 \times$ PBS for $15 \mathrm{~min}$ and fixed in $4 \%$ paraformaldehyde for $10 \mathrm{~min}$ at room temperature. Slides were then washed 3 times in $1 \times$ PBS for 5 min each. Next, slides were incubated in Bouins' solution (5\% acetic acid, $9 \%$ formaldehyde, and $0.9 \%$ picric acid) at room temperature overnight. On the next day, after washing by $1 \times$ PBS, slides were incubated in 0.1\% Fast Green (Fisher, F-99) for 5 min, then in $0.1 \%$ Sirius Red (Direct red 80, Sigma, 0-03035) for $3 \mathrm{~min}$. After 3 times washing in $1 \times$ PBS, slides were dehydrated with ethanol and xylene. Before microscopy, slides were mounted with Neutral Balsam Mounting Medium (Sangon Biotech, E675007).

Injury models. For liver $\mathrm{CCl}_{4}$ injury: $\mathrm{CCl}_{4}$ (SINOPHARM, 10006418) was dissolved at 1:1 in corn oil and administered by gavage at a single dose of $3.5 \mathrm{ml} / \mathrm{kg}$ body weight ${ }^{16}$. For the $\mathrm{CCl}_{4}$-induced chronic injury model, $\mathrm{CCl}_{4}$ was dissolved at 1:3 in corn oil and administrated intragastrically at a dose of $4 \mu \mathrm{l} / \mathrm{g}$ body weight every 3 days, repeated 10 times. For the control groups, mice received the equivalent amount of corn oil.

For liver heat injury (HI): Sterile inflammation induced by thermal injury in the liver was performed as described previously ${ }^{60}$. In detail, mice were anesthetized with $2 \%$ isoflurane gas in a sealed chamber. The abdominal fur was removed carefully and the skin was disinfected with iodine. A midline abdominal incision was made just below the level of the diaphragm to expose the liver. A single focal injury was generated on the surface of the liver with High-Temperature Cautery (WORLD PRECISION INSTRUMENTS, 500392). After that, the skin incisions were closed with suture. For sham groups, mice underwent the same surgical procedure without thermal injury.

For liver cryoinjury (CI): The general surgical process was similar with the liver $\mathrm{HI}$ model. After mice were anesthetized with isoflurane, the liver was exposed and damaged by touching with a copper probe precooled in liquid nitrogen for about $20 \mathrm{~s}$. The control mice were treated with the same procedures as in the CI model but without CI.

For liver acetaminophen (APAP) injury: Food was withdrawn $12-15 \mathrm{~h}$ prior to treatment with APAP (MCE, HY-66005/CS-2819). The drug was administered by gavage with $300 \mathrm{mg} / \mathrm{kg}$ and mice were $\mathrm{CO}_{2}$ euthanized $24 \mathrm{~h}$ later.

For lung bleomycin injury: Alveolar injury was achieved by intratracheal instillation of bleomycin, as described previously ${ }^{61}$. In detail, bleomycin (Sigma B8416) was dissolved at a concentration of $10 \mathrm{U} / \mathrm{ml}$ in sterile PBS (Invitrogen, 10010049) and stored as small aliquots at $-80^{\circ} \mathrm{C}$. Before use, the bleomycin was diluted to a working concentration $1 \mathrm{U} / \mathrm{ml}$ with PBS. After mice were anesthetized with $1 \%$ pentobarbital 
sodium, $2 \mathrm{U} / \mathrm{kg}$ bleomycin was pipetted into the cannula. As the mice breathed bleomycin was inhaled into the lung. The control mice were treated with PBS.

For lung CI: We performed the mice lung CI model according to our lab's protocol as described previously ${ }^{54}$. In detail, mice were first anesthetized with isoflurane in an airtight environment. Then mice were ventilated and anesthetized via tracheotomy, with the cannula connected to a respiratory machine (Harvard Apparatus) and an anesthetic gas machine (Harvard Apparatus). The left lung was exposed by thoracotomy and damaged by touching with a copper probe precooled in liquid nitrogen for about $10 \mathrm{~s}$. After surgery, mice were kept on the warm bed until they awoke. Sham-operated lungs were only exposed without probe application.

For lung LPS injury: $800 \mu \mathrm{g}$ LPS (Escherichia coli 055:B5; Sigma) was dissolved in $50 \mu \mathrm{l}$ of sterile PBS (Invitrogen, 10010049). After mice were anesthetized with $1 \%$ pentobarbital sodium, LPS was pipetted into the cannula by intratracheal instillation. The control mice were treated with $\mathrm{PBS}^{62}$.

Peritoneal macrophages transfer. For peritoneal macrophages transfer, $3 \times 10^{6}$ whole peritoneal cells from G6Mø-CreER;R26-tdTomato mice were transferred intraperitoneally one day prior to $\mathrm{HI}$ and $\mathrm{CCl}_{4}$ treatment as described previously ${ }^{16,17}$. In detail, the peritoneal cells from G6Mø-CreER;R26-tdTomato mice were washed three times with cold $1 \times$ PBS. Then the cells were resuspended with $100 \mu \mathrm{l}$ PBS and transferred to recipients.

Macrophage depletion. Clodronate liposome (YEASEN, Shanghai, China, from Vrije University, Amsterdam) was injected intraperitoneally 7 days prior to the experiment with $200 \mu \mathrm{l} / \mathrm{mice}$.

Detection of serum transaminases ALT and AST. The ALT and AST levels in serum were measured by a kit according to the manufacturer's protocol (Shensuoyoufu Medical Diagnostic company, Shanghai, China) and analyzed by Infinite M200 Pro (TECAN).

In vitro analysis of macrophage-mediated phagocytosis. Pleural and peritoneal cells were cultured in 12-well plate at a suitable density for at least $2 \mathrm{~h}$ to allow adherence. Latex beads-FITC (Sigma, L1030; cell number: beads $=1: 50$ ) was washed 3 times with PBS and finally incubated with FBS at $37^{\circ} \mathrm{C}$ for $1 \mathrm{~h}$ at a concentration of $1 \mu$ l beads: $100 \mu \mathrm{l}$ FBS. At the same time, changed the cell culture medium was changed to FBS-free medium for $1 \mathrm{~h}$ at $37^{\circ} \mathrm{C}$. After incubation, the beads were centrifuged at $10,656 \mathrm{~g}$ for 3 min and resuspended with an FBS-free medium. Next, the beads were added to each well equally and analyzed at the indicated time point by IF and FACS.

In vitro analysis of macrophage polarization. Pleural and peritoneal cells were isolated from mice and cultured in a 12 -well plate at $37^{\circ} \mathrm{C}$ in the presence of $5 \% \mathrm{CO}_{2}$ for $3 \mathrm{~h}$. Then, cell polarization into M1 or M2 was performed by stimulation with IFN- $\gamma$ ( $50 \mathrm{ng} / \mathrm{ml}$, Peprotech, 315-05) plus LPS (10 ng/ml, Solarbio, L8880) or IL4 $(20 \mathrm{ng} / \mathrm{ml}$, Solarbio, P00021), respectively. After $24 \mathrm{~h}$ culture, cells were prepared for RNA extraction and qRT-PCR was done to measure the related gene expression level.

RNA extraction. Cells were harvested for total RNA isolation using Trizol reagent (Invitrogen, 15596018). $1 \mathrm{ml}$ Trizol reagent was used to dissolve each sample and was incubated for 5 mins at room temperature (RT). The samples were centrifuged at $10,656 \mathrm{~g}$ for $5 \mathrm{~min}$ at $4{ }^{\circ} \mathrm{C}$, and the supernatant was transferred to a new $1.5 \mathrm{ml}$ Eppendorf tube followed by adding $200 \mu \mathrm{l}$ chloroform. Then the samples were intensely vortexed for 15-20 s and left for $15 \mathrm{~min}$ at RT. They were then centrifuged at $4^{\circ} \mathrm{C}$ for $10,656 \mathrm{~g}$ for $15 \mathrm{~min}$ and the supernatant transferred to a new 1.5 $\mathrm{ml}$ Eppendorf tube. Next, $500 \mu \mathrm{l}$ of isopropyl alcohol was added and the samples were mixed well and then left to stand at RT for $10 \mathrm{~min}$. Next, the samples were centrifuged at $10,656 \mathrm{~g}$ for $15 \mathrm{~min}$ at $4{ }^{\circ} \mathrm{C}$ and washed RNA with $75 \% \mathrm{EtOH}$ twice, then air-dried. Appropriate RNase/DNase-free water was used to solve the RNA.

Quantitative RT-PCR assay. $1 \mu \mathrm{g}$ total RNA was reverse transcribed to cDNA by TaKaRa PrimeScript ${ }^{\text {mit }}$ RT reagent Kit with gDNA Eraser (Perfect Real Time, Takara, RP074A). Two separate steps followed: first, $42^{\circ} \mathrm{C} 2 \mathrm{~min}$ for Genomic DNA elimination reaction; Second, $37^{\circ} \mathrm{C}$ for $15 \mathrm{~min}, 85^{\circ} \mathrm{C}$ for $5 \mathrm{~s}$ and hold at $4{ }^{\circ} \mathrm{C}$ in PCR machine for reverse-transcription reaction. The synthesized cDNA was diluted fivefold with MilliQ water and then stored at $-20^{\circ} \mathrm{C}$ for further experiments. Quantitative real-time PCR (qRT-PCR) was carried out in ABI Step-one plus instrument (Applied Biosystems) using Invitrogen SYBR Green qRT-PCR reagents (Invitrogen, 4367659). Totally, $10 \mu \mathrm{l}$ reaction system was set up including $5 \mu \mathrm{l} 2 \times$ SYBR Green reagent, $1 \mu \mathrm{l}$ forward and reverse mixed primers, $1 \mu$ diluted

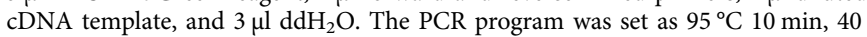
repetitions of $95^{\circ} \mathrm{C} 5 \mathrm{~s}$ and $60^{\circ} \mathrm{C} 30 \mathrm{~s}$. The results were normalized to GAPDH and control was set as 1. All primers are listed in Supplementary Table 1.

Quantification and statistical analysis. All results were presented as the mean \pm SD. Statistical analyses were performed using Student's $t$ test with GraphPad Prism 7 software. Totally, 4-8 mice were included in each group of mice in every experiment.
Multiple groups were tested via one-way ANOVA, and comparisons between two groups were performed using Student's $t$ test. ${ }^{*} P$ value of $<0.05$, ${ }^{* *} P$ value of $<0.01$, ${ }^{* * *} P$ value of $<0.005$, and ${ }^{* * * *} P$ value of $<0.0001$ were considered significant.

Reporting summary. Further information on research design is available in the Nature Research Reporting Summary linked to this article.

\section{Data availability}

All data that support the findings of this study are provided within the paper and its supplementary information. All additional information is available from the corresponding author upon reasonable request. Source data are provided with this paper.

Received: 10 September 2020; Accepted: 15 April 2021; Published online: 17 May 2021

\section{References}

1. Geissmann, F. et al. Development of monocytes, macrophages, and dendritic cells. Science 327, 656-661 (2010).

2. Wynn, T. A., Chawla, A. \& Pollard, J. W. Macrophage biology in development homeostasis and disease. Nature 496, 445-455 (2013).

3. van Furth, R. \& Cohn, Z. A. The origin and kinetics of mononuclear phagocytes. J. Exp. Med. 128, 415-435 (1968).

4. van Furth, R. \& Diesselhoff-Den Dulk, M. M. The kinetics of promonocytes and monocytes in the bone marrow. J. Exp. Med. 132, 813-828 (1970).

5. Serhan, C. N. et al. Resolution of inflammation: state of the art, definitions and terms. FASEB J. 21, 325-332 (2007).

6. Heidt, T. et al. Differential contribution of monocytes to heart macrophages in steady-state and after myocardial infarction. Circ. Res. 115, 284-295 (2014).

7. Ginhoux, F. et al. Fate mapping analysis reveals that adult microglia derive from primitive macrophages. Science 330, 841-845 (2010).

8. Hashimoto, D. et al. Tissue-resident macrophages self-maintain locally throughout adult life with minimal contribution from circulating monocytes. Immunity 38, 792-804 (2013)

9. Epelman, S., Lavine, K. J. \& Randolph, G. J. Origin and functions of tissue macrophages. Immunity 41, 21-35 (2014).

10. Perdiguero, E. G. \& Geissmann, F. The development and maintenance of resident macrophages. Nat. Immunol. 17, 2-8 (2016).

11. Lavine, K. J. et al. Distinct macrophage lineages contribute to disparate patterns of cardiac recovery and remodeling in the neonatal and adult heart. Proc. Natl Acad. Sci. USA 111, 16029-16034 (2014).

12. Epelman, S. et al. Embryonic and adult-derived resident cardiac macrophages are maintained through distinct mechanisms at steady state and during inflammation. Immunity 40, 91-104 (2014).

13. Dutta, P. et al. Myocardial infarction activates CCR2 $(+)$ hematopoietic stem and progenitor cells. Cell Stem Cell 16, 477-487 (2015).

14. Bajpai, G. et al. The human heart contains distinct macrophage subsets with divergent origins and functions. Nat. Med. 24, 1234-1245 (2018).

15. Dick, S. A. et al. Self-renewing resident cardiac macrophages limit adverse remodeling following myocardial infarction. Nat. Immunol. 20, 29-39 (2019).

16. Wang, J. \& Kubes, P. A reservoir of mature cavity macrophages that can rapidly invade visceral organs to affect tissue repair. Cell 165, 668-678 (2016).

17. Deniset, J. F. et al. Gata6 ${ }^{+}$pericardial cavity macrophages relocate to the injured heart and prevent cardiac fibrosis. Immunity 51, 131-140.e5 (2019).

18. Okabe, Y. \& Medzhitov, R. Wormhole travel for macrophages. Cell $\mathbf{1 6 5}$ 518-519 (2016).

19. Okabe, Y. \& Medzhitov, R. Tissue-specific signals control reversible program of localization and functional polarization of macrophages. Cell 157, 832-844 (2014)

20. Rosas, M. et al. The transcription factor Gata6 links tissue macrophage phenotype and proliferative renewal. Science 344, 645-648 (2014).

21. Gautier, E. L. et al. Gene-expression profiles and transcriptional regulatory pathways that underlie the identity and diversity of mouse tissue macrophages. Nat. Immunol. 13, 1118-1128 (2012).

22. Gautier, E. L. et al. Gata6 regulates aspartoacylase expression in resident peritoneal macrophages and controls their survival. J. Exp. Med. 211, 1525-1531 (2014).

23. Zheng, R. et al. Function of GATA factors in the adult mouse liver. PLoS ONE 8, e83723 (2013).

24. Prendiville, T. W. et al. Novel roles of GATA4/6 in the postnatal heart identified through temporally controlled, cardiomyocyte-specific gene inactivation by adeno-associated virus delivery of Cre recombinase. PLOS ONE 10, e0128105 (2015).

25. Cheung, W. K. et al. Control of alveolar differentiation by the lineage transcription factors GATA6 and HOPX inhibits lung adenocarcinoma metastasis. Cancer Cell 23, 725-738 (2013). 
26. Almalki, S. G. \& Agrawal, D. K. Key transcription factors in the differentiation of mesenchymal stem cells. Differentiation 92, 41-51 (2016).

27. Anastassiadis, K. et al. Dre recombinase, like Cre, is a highly efficient sitespecific recombinase in E. coli, mammalian cells and mice. Dis. Model. Mech. 2, 508-515 (2009).

28. Hermann, M. et al. Binary recombinase systems for high-resolution conditional mutagenesis. Nucleic Acids Res. 42, 3894-3907 (2014).

29. He, L. et al. Genetic lineage tracing of resident stem cells by DeaLT. Nat. Protoc. 13, 2217-2246 (2018)

30. $\mathrm{Pu}, \mathrm{W}$. et al. Genetic targeting of organ-specific blood vessels. Circ. Res. 123, 86-99 (2018).

31. Zhang, H. et al. Genetic lineage tracing identifies endocardial origin of liver vasculature. Nat. Genet. 48, 537-543 (2016).

32. He, L. et al. Enhancing the precision of genetic lineage tracing using dual recombinases. Nat. Med. 23, 1488-1498 (2017).

33. Madisen, L. et al. A robust and high-throughput Cre reporting and characterization system for the whole mouse brain. Nat. Neurosci. 13, 133-140 (2010).

34. de Meijer, V. E. et al. Broad-spectrum matrix metalloproteinase inhibition curbs inflammation and liver injury but aggravates experimental liver fibrosis in mice. PLoS ONE 5, e11256 (2010).

35. Buch, T. et al. A Cre-inducible diphtheria toxin receptor mediates cell lineage ablation after toxin administration. Nat. Methods 2, 419-426 (2005).

36. Naglich, J. G., Metherall, J. E., Russell, D. W. \& Eidels, L. Expression cloning of a diphtheria toxin receptor: identity with a heparin-binding EGF-like growth factor precursor. Cell 69, 1051-1061 (1992).

37. Tang, J. et al. Arterial Sca1+ vascular stem cells generate de novo smooth muscle for artery repair and regeneration. Cell Stem Cell 26, 81-96.e4 (2020).

38. Liu, Z. et al. Fate mapping via Ms4a3-expression history traces monocytederived cells. Cell 178, 1509-1525.e19 (2019).

39. Stern, C. D. \& Fraser, S. E. Tracing the lineage of tracing cell lineages. Nat. Cell Biol. 3, E216-E218 (2001).

40. Dor, Y., Brown, J., Martinez, O. I. \& Melton, D. A. Adult pancreatic beta-cells are formed by self-duplication rather than stem-cell differentiation. Nature 429, 41-46 (2004).

41. Barker, N. et al. Identification of stem cells in small intestine and colon by marker gene Lgr5. Nature 449, 1003-1007 (2007).

42. Samokhvalov, I. M., Samokhvalova, N. I. \& Nishikawa, S. Cell tracing shows the contribution of the yolk sac to adult haematopoiesis. Nature 446, 1056-1061 (2007).

43. Kretzschmar, K. \& Watt, F. M. Lineage tracing. Cell 148, 33-45 (2012).

44. Eschenhagen, T. et al. Cardiomyocyte regeneration: a consensus statement. Circulation 136, 680-686 (2017).

45. Yona, S. et al. Fate mapping reveals origins and dynamics of monocytes and tissue macrophages under homeostasis. Immunity 38, 79-91 (2013).

46. Hoeffel, G. et al. C-Myb(+) erythro-myeloid progenitor-derived fetal monocytes give rise to adult tissue-resident macrophages. Immunity 42, 665-678 (2015).

47. Ginhoux, F. \& Guilliams, M. Tissue-resident macrophage ontogeny and homeostasis. Immunity 44, 439-449 (2016).

48. Blanpain, C. \& Fuchs, E. Epidermal homeostasis: a balancing act of stem cells in the skin. Nat. Rev. Mol. Cell Biol. 10, 207-217 (2009).

49. Kondo, M. et al. Biology of hematopoietic stem cells and progenitors: implications for clinical application. Annu. Rev. Immunol. 21, 759-806 (2003).

50. Sun, J. et al. Clonal dynamics of native haematopoiesis. Nature 514, 322-327 (2014).

51. Kordon, E. C. \& Smith, G. H. An entire functional mammary gland may comprise the progeny from a single cell. Development 125, 1921-1930 (1998).

52. Van Keymeulen, A. et al. Distinct stem cells contribute to mammary gland development and maintenance. Nature 479, 189-193 (2011).

53. Kajstura, J. et al. Evidence for human lung stem cells. N. Engl. J. Med. 364 1795-1806 (2011).

54. Liu, Q. et al. c-kit(+) cells adopt vascular endothelial but not epithelial cell fates during lung maintenance and repair. Nat. Med. 21, 866-868 (2015).

55. Madisen, L. et al. A toolbox of Cre-dependent optogenetic transgenic mice for light-induced activation and silencing. Nat. Neurosci. 15, 793-802 (2012).

56. Zhang, H. et al. Endocardium minimally contributes to coronary endothelium in the embryonic ventricular free walls. Circ. Res. 118, 1880-1893 (2016)

57. Sodhi, C. P., Li, J. \& Duncan, S. A. Generation of mice harbouring a conditional loss-of-function allele of Gata6. BMC Dev. Biol. 6, 19 (2006).

58. Ghosn, E. E. et al. Two physically, functionally, and developmentally distinct peritoneal macrophage subsets. Proc. Natl Acad. Sci. USA 107, 2568-2573 (2010).

59. Yu, W. et al. GATA4 regulates Fgf16 to promote heart repair after injury. Development 143, 936-949 (2016).
60. Dal-Secco, D. et al. A dynamic spectrum of monocytes arising from the in situ reprogramming of CCR2 + monocytes at a site of sterile injury. J. Exp. Med. 212, 447-456 (2015).

61. Liu, Q. et al. Lung regeneration by multipotent stem cells residing at the bronchioalveolar-duct junction. Nat. Genet. 51, 728-738 (2019).

62. Mei, S. H. et al. Prevention of LPS-induced acute lung injury in mice by mesenchymal stem cells overexpressing angiopoietin 1. PLoS Med. 4, e269 (2007)

\section{Acknowledgements}

This study was supported by the National Key Research \& Development Program of China (2019YFA0110403, 2019YFA0802000, 2018YFA0108100, 2018YFA0107900, 2019YFA0802803, and 2020YFA0803202), National Science Foundation of China (8208810001, 31730112, 31625019, 91849202, 31922032, 81872241, 31900625, 32050087, 32070727, and 31801215), Strategic Priority Research Program of the Chinese Academy of Sciences (CAS, XDA16010507 and XDB19000000), Key Project of Frontier Sciences of CAS (QYZDB-SSW-SMC003), Shanghai Science and Technology Commission (19JC1415700, 19YF1455300, 19ZR1479800, 20QC1401000, and 18YF1427600), Collaborative Innovation Program of Shanghai Municipal Health Commission (2020CXJQ01), the Pearl River Talent Recruitment Program of Guangdong Province (2017ZT07S347), Young Elite Scientists Sponsorship Program Grant of CAS, Youth Innovation Promotion Association Grant, Shanghai Yangfan Project, Shanghai Rising Star Project, China Postdoctoral Science Foundation, China Postdoctoral Innovative Talent Support Program, Royal Society-Newton Advanced Fellowship, AstraZeneca, and Sanofi-SIBS Fellowship, Boehringer-Ingelheim, and the support from the XPLORER PRIZE. We thank Shanghai Model Organisms Center, Inc. for mouse generation. We thank Dr. Hongkui Zeng for kindly providing reporter mice and Dr. Yi Zeng for kindly providing the Gata $^{\text {flox }}$ mouse (jax008196). We thank members of the animal facility and cell platform in CEMCS, and the National Center for Protein Science Shanghai for assistance in microscopy.

\section{Author contributions}

H.J., K.L., and B.Z. designed the study, performed experiments, and analyzed the data. J T., X.H., H.W., Q.Z., H. Zhu, Yan Li, W.P., H. Zhao, L.H., Yi Li, S.Z., Z.Z., and Y.Z. bred the mice, performed the experiments, and provided the intellectual input. Y.Q. offered assistance in microscopy. S.P., K.K., and W.Z. supported the study. F.G. and Z.L. provided the Ms4a3-CreER mouse strain. Y.J., B.H., and L.W. provided intellectual input, edited the paper, or supported important reagents. B.Z. conceived and supervised the study, and wrote the paper.

\section{Competing interests}

The authors declare no competing interests.

\section{Additional information}

Supplementary information The online version contains supplementary material available at https://doi.org/10.1038/s41467-021-23197-7.

Correspondence and requests for materials should be addressed to B.Z.

Peer review information Nature Communications thanks Martin Guilliams, Paul Kaye, and Frank Tacke for their contribution to the peer review of this work.

Reprints and permission information is available at http://www.nature.com/reprints

Publisher's note Springer Nature remains neutral with regard to jurisdictional claims in published maps and institutional affiliations.

Open Access This article is licensed under a Creative Commons Attribution 4.0 International License, which permits use, sharing, adaptation, distribution and reproduction in any medium or format, as long as you give appropriate credit to the original author(s) and the source, provide a link to the Creative Commons license, and indicate if changes were made. The images or other third party material in this article are included in the article's Creative Commons license, unless indicated otherwise in a credit line to the material. If material is not included in the article's Creative Commons license and your intended use is not permitted by statutory regulation or exceeds the permitted use, you will need to obtain permission directly from the copyright holder. To view a copy of this license, visit http://creativecommons.org/ licenses/by/4.0/

(c) The Author(s) 2021 